\title{
Local properties of Richardson varieties in the Grassmannian via a bounded Robinson-Schensted-Knuth correspondence
}

\author{
Victor Kreiman
}

Received: 17 May 2006 / Accepted: 6 August 2007 / Published online: 18 September 2007

(C) Springer Science+Business Media, LLC 2007

\begin{abstract}
The Richardson variety $X_{\alpha}^{\gamma}$ in the Grassmannian is defined to be the intersection of the Schubert variety $X^{\gamma}$ and opposite Schubert variety $X_{\alpha}$. We give an explicit Gröbner basis for the ideal of the tangent cone at any $T$-fixed point of $X_{\alpha}^{\gamma}$, thus generalizing a result of Kodiyalam-Raghavan (J. Algebra 270(1):28-54, 2003) and Kreiman-Lakshmibai (Algebra, Arithmetic and Geometry with Applications, 2004). Our proof is based on a generalization of the Robinson-Schensted-Knuth (RSK) correspondence, which we call the bounded RSK (BRSK). We use the Gröbner basis result to deduce a formula which computes the multiplicity of $X_{\alpha}^{\gamma}$ at any $T$-fixed point by counting families of nonintersecting lattice paths, thus generalizing a result first proved by Krattenthaler (Sém. Lothar. Comb. 45:B45c, 2000/2001; J. Algebr. Comb. 22:273-288, 2005).
\end{abstract}

Keywords Schubert variety · Grassmannian · Multiplicity · Grobner basis · Robinson-Schensted-Knuth correspondence

\section{Introduction}

The Richardson variety $X_{\alpha}^{\gamma}$ in the Grassmannian ${ }^{1}$ is defined to be the intersection of the Schubert variety $X^{\gamma}$ and opposite Schubert variety $X_{\alpha}$. In particular, Schubert and opposite Schubert varieties are special cases of Richardson varieties. We derive local properties of $X_{\alpha}^{\gamma}$ at any $T$-fixed point $e_{\beta}$. It should be noted that the local properties of Schubert varieties at $T$-fixed points determine their local properties at all other

\footnotetext{
V. Kreiman ( $₫)$

Department of Mathematics, University of Georgia, Athens, GA 30602, USA

e-mail: vkreiman@math.uga.edu

${ }^{1}$ Richardson varieties in the Grassmannian are also studied by Stanley in [20], where these varieties are called skew Schubert varieties. Discussion of these varieties also appears in [6].
} 
points, because of the $B$-action; but this does not extend to Richardson varieties, since Richardson varieties only have a $T$-action.

In Kodiyalam-Raghavan [7] and Kreiman-Lakshmibai [11], an explicit Gröbner basis for the ideal of the tangent cone of the Schubert variety $X^{\gamma}$ at $e_{\beta}$ is obtained. The Gröbner basis is used to derive a formula for the multiplicity of $X^{\gamma}$ at $e_{\beta}$. In this paper, we generalize the results of [7] and [11] to the case of Richardson varieties. The results of [7] and [11] were conjectured by Kreiman-Lakshmibai [12], although in a different, group-theoretic form. The multiplicity formula (in both forms) was first proved by Krattenthaler [8, 9] by showing its equivalence to the RosenthalZelevinsky determinantal multiplicity formula [18].

Sturmfels [21] and Herzog-Trung [5] proved results on a class of determinantal varieties which are equivalent to the results of [7,11], and this paper for the case of Schubert varieties at the $T$-fixed point $e_{i d}$. The key to their proofs was to use a version of the Robinson-Schensted-Knuth correspondence (which we shall call the 'ordinary' RSK) in order to establish a degree-preserving bijection between a set of monomials defined by an initial ideal and a 'standard monomial basis'.

The difficulty in generalizing this method of proof to the case of Schubert varieties at an arbitrary $T$-fixed point $e_{\beta}$ lies in generalizing this bijection. All three of [7, 11], and this paper obtain generalizations of this bijection. The three generalizations, when restricted to Schubert varieties, are in fact the same bijection ${ }^{2}$, although this is not immediately apparent. Although the formulations of the bijection in [7] and [11] are similar to eachother, our formulation is in terms of different combinatorial indexing sets, and thus most of our combinatorial definitions and proofs are of a different nature than those of [7] and [11]. The relationship between our formulation and the formulations in [7] and [11] is analogous to the relationship between the RobinsonSchensted correspondence and Viennot's version of the Robinson-Schensted correspondence [19, 22].

We formulate the bijection by introducing a generalization of the ordinary RSK, which we call the bounded RSK. Because the definition of the bounded RSK is built from that of the ordinary RSK, many properties of the bounded RSK are immediate consequences of analogous properties for the ordinary RSK (see [3, 19]). This simplifies our proofs.

Results analogous to those of [7] and [11] have now been obtained for the symplectic and orthogonal Grassmannians (see $[4,16]$ ). We believe it is possible that the methods of this paper can be adapted to these varieties as well. The results of $[7,11]$, and this paper have been used to study the equivariant cohomology and equivariant K-theory of the Grassmannian (see [10, 15]).

\section{Statement of results}

Let $K$ be an algebraically closed field, and let $d, n$ be fixed positive integers, $0<d<$ $n$. The Grassmannian $G r_{d, n}$ is the set of all $d$-dimensional subspaces of $K^{n}$. The

\footnotetext{
${ }^{2}$ This supports the conviction of the authors in [7] that this bijection is natural and that it is in some sense the only natural bijection satisfying the required geometric conditions.
} 
Plücker map $\mathrm{pl}: G r_{d, n} \rightarrow \mathbb{P}\left(\wedge^{d} K^{n}\right)$ is given by $\operatorname{pl}(W)=\left[w_{1} \wedge \cdots \wedge w_{d}\right]$, where $\left\{w_{1}, \ldots, w_{d}\right\}$ is any basis for $W$. It is well known that $\mathrm{pl}$ is a bijection onto a closed subset of $\mathbb{P}\left(\wedge^{d} K^{n}\right)$. Thus $G r_{d, n}$ inherits the structure of a projective variety.

Define $I_{d, n}$ to be the set of $d$-element subsets of $\{1, \ldots, n\}$. Let $\alpha=\left\{\alpha_{1}, \ldots, \alpha_{d}\right\} \in$ $I_{d, n}, \alpha_{1}<\cdots<\alpha_{d}$. Define the complement of $\alpha$ by $\bar{\alpha}=\{1, \ldots, n\} \backslash \alpha$, and the length of $\alpha$ by $l(\alpha)=\alpha_{1}+\cdots+\alpha_{d}-\left(\begin{array}{c}d+1 \\ 2\end{array}\right)$. If $\beta=\left\{\beta_{1}, \ldots, \beta_{d}\right\} \in I_{d, n}, \beta_{1}<\ldots<$ $\beta_{d}$, then we say that $\alpha \leq \beta$ if $\alpha_{i} \leq \beta_{i}, i=1, \ldots, d$.

Let $\left\{e_{1}, \ldots, e_{n}\right\}$ be the standard basis for $K^{n}$. For $\alpha \in I_{d, n}$, define $e_{\alpha}=$ $\operatorname{Span}\left\{e_{\alpha_{1}}, \ldots, e_{\alpha_{d}}\right\} \in G r_{d, n}$. Let

$$
\begin{aligned}
& G=G L_{n}(K), \\
& B=\{g \in G \mid g \text { is upper triangular }\}, \\
& B^{-}=\{g \in G \mid g \text { is lower triangular }\}, \\
& T=\{g \in G \mid g \text { is diagonal }\} .
\end{aligned}
$$

The group $G$ acts transitively on $G r_{d, n}$ with $T$-fixed points $\left\{e_{\alpha} \mid \alpha \in I_{d, n}\right\}$. The Zariski closure of the $B$ (resp. $B^{-}$) orbit through $e_{\alpha}$, with canonical reduced scheme structure, is called a Schubert variety (resp. opposite Schubert variety), and denoted by $X^{\alpha}$ (resp. $X_{\alpha}$ ). For $\alpha, \gamma \in I_{d, n}$, the scheme-theoretic intersection $X_{\alpha}^{\gamma}=$ $X_{\alpha} \cap X^{\gamma}$ is called a Richardson variety. It can be shown that $X_{\alpha}^{\gamma}$ is nonempty if and only if $\alpha \leq \gamma$; that $e_{\beta} \in X_{\alpha}^{\gamma}$ if and only if $\alpha \leq \beta \leq \gamma$; and that if $X_{\alpha}^{\gamma}$ is nonempty, it is reduced and irreducible of dimension $l(\gamma)-l(\alpha)$ (see $[1,13,14,17])$.

For $\beta \in I_{d, n}$ define $p_{\beta}$ to be homogeneous (Plücker) coordinate $\left[e_{\beta_{1}} \wedge \cdots \wedge e_{\beta_{d}}\right]^{*}$ of $\mathbb{P}\left(\wedge^{d} K^{n}\right)$. Let $\mathcal{O}_{\beta}$ be the distinguished open set of $G r_{d, n}$ defined by $p_{\beta} \neq 0$. Its coordinate ring $K\left[\mathcal{O}_{\beta}\right]$ is isomorphic to the homogeneous localization $K\left[G r_{d, n}\right]_{\left(p_{\beta}\right)}$. Define $f_{\theta, \beta}$ to be $p_{\theta} / p_{\beta} \in K\left[\mathcal{O}_{\beta}\right]$.

The open set $\mathcal{O}_{\beta}$ is isomorphic to the affine space $K^{d(n-d)}$. Indeed, it can be identified with the space of matrices in $M_{n \times d}$ in which rows $\beta_{1}, \ldots, \beta_{d}$ are the rows of the $d \times d$ identity matrix, and rows $\bar{\beta}_{1}, \ldots, \bar{\beta}_{n-d}$ contain arbitrary elements of $K$. The rows of $\mathcal{O}_{\beta}$ are indexed by $\{1, \ldots, n\}$, and the columns by $\beta$. Note that the affine coordinates of $K\left[\mathcal{O}_{\beta}\right]$ are indexed by $\bar{\beta} \times \beta$. The coordinate $f_{\theta, \beta}, \theta \in I_{d, n}$, is identified with plus or minus the $d \times d$ minor of $\mathcal{O}_{\beta}$ with row-set $\theta_{1}, \ldots, \theta_{d}$.

Example 2.1 Let $d=3, n=7, \beta=\{2,5,7\}$. Then

$$
\mathcal{O}_{\beta}=\left\{\left(\begin{array}{ccc}
x_{12} & x_{15} & x_{17} \\
1 & 0 & 0 \\
x_{32} & x_{35} & x_{37} \\
x_{42} & x_{45} & x_{47} \\
0 & 1 & 0 \\
x_{62} & x_{65} & x_{67} \\
0 & 0 & 1
\end{array}\right), x_{i j} \in K\right\},
$$

and

$$
f_{\{1,4,5\}, \beta}=\left|\begin{array}{ccc}
x_{12} & x_{15} & x_{17} \\
x_{42} & x_{45} & x_{47} \\
0 & 1 & 0
\end{array}\right|=-\left|\begin{array}{ll}
x_{12} & x_{17} \\
x_{42} & x_{47}
\end{array}\right| .
$$


In order to better understand the local properties of $X_{\alpha}^{\gamma}$ near $e_{\beta}$, we analyze $Y_{\alpha, \beta}^{\gamma}=$ $X_{\alpha}^{\gamma} \cap \mathcal{O}_{\beta}$, an open subset of $X_{\alpha}^{\gamma}$ centered at $e_{\beta}$, and a closed affine subvariety of $\mathcal{O}_{\beta}$. Let $G_{\alpha, \beta}^{\gamma}=\left\{f_{\theta, \beta} \mid \alpha \nsucceq \theta\right.$ or $\left.\theta \not z \gamma\right\} \subset K\left[\mathcal{O}_{\beta}\right]$, and let $\left\langle G_{\alpha, \beta}^{\gamma}\right\rangle$ be the ideal of $K\left[\mathcal{O}_{\beta}\right]$ generated by $G_{\alpha, \beta}^{\gamma}$. The following is a well known result (see $[1,14]$, for instance).

Theorem 2.2 $K\left[Y_{\alpha, \beta}^{\gamma}\right]=K\left[\mathcal{O}_{\beta}\right] /\left\langle G_{\alpha, \beta}^{\gamma}\right\rangle$.

As a consequence of Theorem 2.2, $Y_{\alpha, \beta}^{\gamma}$ is isomorphic to the tangent cone of $X_{\alpha}^{\gamma}$ at $e_{\beta}$, and thus $\operatorname{deg} Y_{\alpha, \beta}^{\gamma}=$ Mult $_{e_{\beta}} X_{\alpha}^{\gamma}$, the multiplicity of $X_{\alpha}^{\gamma}$ at $e_{\beta}$. Indeed, since $Y_{\alpha, \beta}^{\gamma}$ is an affine variety in $\mathcal{O}_{\beta}$ defined by a homogeneous ideal, with $e_{\beta}$ the origin of $\mathcal{O}_{\beta}$, $Y_{\alpha, \beta}^{\gamma}$ is isomorphic to the tangent cone of $Y_{\alpha, \beta}^{\gamma}$ at $e_{\beta}$; since $Y_{\alpha, \beta}^{\gamma}$ is open in $X_{\alpha}^{\gamma}$, the tangent cone of $Y_{\alpha, \beta}^{\gamma}$ at $e_{\beta}$ is isomorphic to the tangent cone of $X_{\alpha}^{\gamma}$ at $e_{\beta}$.

Any minor $f_{\theta, \beta}$ can be expressed naturally as plus or minus a determinant all of whose entries are $x_{i j}$ 's. Choose a monomial order on $K\left[\mathcal{O}_{\beta}\right]$ such that the initial term of any minor $f_{\theta, \beta}$, in $\left(f_{\theta, \beta}\right)$, is the Southwest-Northeast diagonal of this determinant. The main result of this paper, which is also proven in [7] and [11], is the following.

Proposition 2.3 $G_{\alpha, \beta}^{\gamma}$ is a Gröbner basis for $\left\langle G_{\alpha, \beta}^{\gamma}\right\rangle$.

If $S$ is any subset of $K\left[\mathcal{O}_{\beta}\right]$, define in $S$ to be the ideal $\langle\operatorname{in}(s) \mid s \in S\rangle$.

Corollary $2.4 \operatorname{deg} Y_{\alpha, \beta}^{\gamma}\left(=\right.$ Mult $\left._{e_{\beta}} X_{\alpha}^{\gamma}\right)$ is the number of square-free monomials of degree $l(\gamma)-l(\alpha)$ in $K\left[\mathcal{O}_{\beta}\right] \backslash$ in $G_{\alpha, \beta}^{\gamma}$.

We now briefly sketch the proof of Proposition 2.3 (omitting the details, which can be found in Section 8), in order to introduce the main combinatorial objects of interest and outline the structure of this paper. We wish to show that in any degree, the number of monomials of in $G_{\alpha, \beta}^{\gamma}$ is at least as great as the number of monomials of in $\left\langle G_{\alpha, \beta}^{\gamma}\right\rangle$ (the other inequality being trivial), or equivalently, that in any degree, the number of monomials of $K\left[\mathcal{O}_{\beta}\right] \backslash$ in $G_{\alpha, \beta}^{\gamma}$ is no greater than the number of monomials of $K\left[\mathcal{O}_{\beta}\right] \backslash$ in $\left\langle G_{\alpha, \beta}^{\gamma}\right\rangle$. Both the monomials of $K\left[\mathcal{O}_{\beta}\right] \backslash$ in $\left\langle G_{\alpha, \beta}^{\gamma}\right\rangle$ and the standard monomials on $Y_{\alpha, \beta}^{\gamma}$ form a basis for $K\left[\mathcal{O}_{\beta}\right] /\left\langle G_{\alpha, \beta}^{\gamma}\right\rangle$, and thus agree in cardinality in any degree. Therefore, it suffices to give a degree-preserving injection from the monomials of $K\left[\mathcal{O}_{\beta}\right] \backslash$ in $G_{\alpha, \beta}^{\gamma}$ to the standard monomials on $Y_{\alpha, \beta}^{\gamma}$. We construct such an injection, the bounded RSK (BRSK), from an indexing set of the former to an indexing set of the latter. These indexing sets are given in Table 1 .

In Sects. 3, 4, 5, 6, and 7, we define nonvanishing multisets on $\bar{\beta} \times \beta$ bounded by $T_{\alpha}, W_{\gamma}$, nonvanishing semistandard notched bitableaux on $\bar{\beta} \times \beta$ bounded by $T_{\alpha}, W_{\gamma}$, and the injection BRSK from the former to the latter. In Section 8, we prove that these two combinatorial objects are indeed indexing sets for the monomials of $K\left[\mathcal{O}_{\beta}\right] \backslash$ in $G_{\alpha, \beta}^{\gamma}$ and the standard monomials on $Y_{\alpha, \beta}^{\gamma}$ respectively, and use this to prove Proposition 2.3 and Corollary 2.4. In Sections 9 and 10, we show how using Corollary 2.4, Mult $e_{\beta} X_{\alpha}^{\gamma}$ can be interpreted as counting certain families of nonintersecting paths in the lattice $\bar{\beta} \times \beta$. In Section 11, we give two of the more detailed proofs. 
Table 1 Two subsets of $K\left[\mathcal{O}_{\beta}\right]$ and their indexing sets

\begin{tabular}{ll}
\hline Set of elements in $K\left[\mathcal{O}_{\beta}\right]$ & Indexing set \\
\hline monomials of $K\left[\mathcal{O}_{\beta}\right] \backslash$ in $G_{\alpha, \beta}^{\gamma}$ & nonvanishing multisets \\
& on $\bar{\beta} \times \beta$ bounded by $T_{\alpha}, W_{\gamma}$ \\
standard monomials on $Y_{\alpha, \beta}^{\gamma}$ & nonvanishing semistandard notched bitableaux \\
& on $\bar{\beta} \times \beta$ bounded by $T_{\alpha}, W_{\gamma}$ \\
\hline
\end{tabular}

\section{Notched tableaux and bounded insertion}

A Young diagram (resp. notched diagram) is a collection of boxes arranged into a left and top justified array (resp. into left justified rows). The empty Young diagram is the Young diagram with no boxes. A notched diagram may contain rows with no boxes; however, a Young diagram may not, unless it is the empty Young diagram. A Young tableau (resp. notched tableau) is a filling of the boxes of a Young diagram (resp. notched diagram) with positive integers. The empty Young tableau is the Young tableau with no boxes. Let $P$ be either a notched tableau or a Young tableau. We denote by $P_{i}$ the $i$-th row of $P$ from the top, and by $P_{i, j}$ the $j$-th entry from the left of $P_{i}$. We say that $P$ is row strict if the entries of any row of $P$ strictly increase as you move to the right. If $P$ is a Young tableau, then we say that $P$ is semistandard if it is row strict and the entries of any column weakly increase as you move down. By definition, the empty Young tableau is considered semistandard.

Example 3.1 A row strict notched tableau $P$, and a semistandard Young tableau $R$.

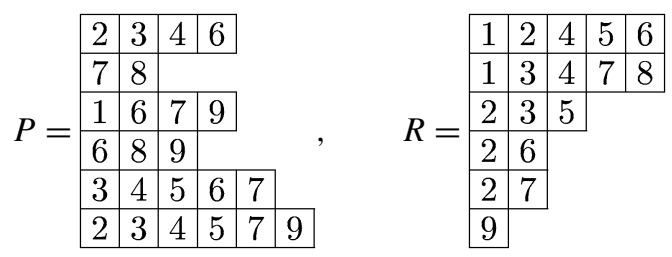

Let $P$ be a row strict notched tableau, and $b$ a positive integer. Since $P$ is row strict, its entries which are greater than or equal to $b$ are right justified in each row. Thus if we remove these entries (and their boxes) from $P$ then we are left with a row strict notched tableau, which we denote by $P^{<b}$. We say that $P$ is semistandard on b if $P^{<b}$ is a semistandard Young tableau (note that if $P$ is semistandard on $b$ and the first row of $P^{<b}$ has no boxes, then $P^{<b}$ must be the empty Young tableau). It is clear that if $P$ is semistandard on $b$, then it is semistandard on $b^{\prime}$ for any positive integer $b^{\prime}<b$. 
Example 3.2 Let

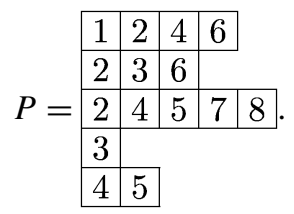

Then

$$
P^{<5}=\begin{array}{l|l|l|l}
\hline 1 & 2 & 4 \\
\hline 2 & 3 & \\
\cline { 1 - 2 } 2 & 4 \\
\cline { 1 - 1 } 3 &
\end{array} \quad, \quad P^{<6}=\begin{array}{l|l|l|l|}
\hline 1 & 2 & 4 \\
\hline 2 & & 3 & \\
\hline 2 & 2 & 4 & 5 \\
\hline 3 & \multicolumn{3}{|l}{} \\
\hline 4 & \multicolumn{2}{|l}{} \\
\hline
\end{array}
$$

Thus $P$ is semistandard on 5 but not on 6 .

We next review the transpose of Schensted's column insertion process, which we shall call simply 'ordinary' Schensted insertion. It is an algorithm which takes as input a semistandard Young tableau $P$ and a positive integer $a$, and produces as output a new semistandard Young tableau with the same shape as $P$ plus one extra box, and with the same entries as $P$ (possibly in different locations) plus one additional entry, namely $a$. To begin, insert $a$ into the first row of $R$, as follows. If $a$ is greater than all entries in the first row of $R$, then place $a$ in a new box on the right end of the first row, and ordinary Schensted insertion terminates. Otherwise, find the smallest entry of the first row of $R$ which is greater than or equal to $a$, and replace that number with $a$. We say that the number which was replaced was "bumped" from the first row. Insert the bumped number into the second row in precisely the same fashion that $a$ was inserted into the first row. This process continues down the rows until, at some point, a number is placed in a new box on the right end of some row, at which point ordinary Schensted insertion terminates.

We next describe the bounded insertion algorithm, which takes as input a positive integer $b$, a notched tableau $P$ which is semistandard on $b$, and a positive integer $a<b$, and produces as output a notched tableau which is semistandard on $b$, which we denote by $P \stackrel{b}{\leftarrow} a$.

\section{Bounded Insertion}

Step 1. Remove all entries of $P$ which are greater than or equal to $b$ from $P$, resulting in the semistandard Young tableau $P^{<b}$.

Step 2. Insert a into $P^{<b}$ using the ordinary Schensted insertion process.

Step 3. Place the entries of $P$ which were removed when forming $P^{<b}$ in Step 1 back into the Young tableau resulting from Step 2, in the same rows from which they were removed.

This insertion process is effectively the ordinary Schensted insertion of $a$ into $P$, but acting only on the part of $P$ which is "bounded" by $b$. The fact that bounded insertion preserves the property of being semistandard on $b$ follows immediately from the fact that ordinary Schensted insertion preserves the property of being semistandard. 
Example 3.3 Let

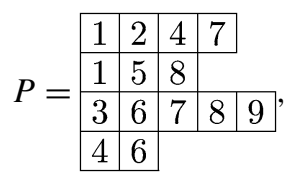

$a=3, b=6$. We compute $P \stackrel{b}{\leftarrow} a$.

Step 1. Remove all entries of $P$ which are greater than or equal than $b$, resulting in

$$
P^{<b}=\begin{array}{|l|l|l|}
\hline 1 & 2 & 4 \\
\hline 1 & 5 & \\
\cline { 1 - 1 } 3 & \multicolumn{2}{|l}{} \\
\cline { 1 - 1 } 4 &
\end{array} .
$$

Step 2. Insert $a$ into $P^{<b}$ using the ordinary Schensted insertion process: $a$ bumps 4 from the first row, which bumps 5 from the second row, which is placed in a new box on the right end of the third row, to form

\begin{tabular}{|l|l|l|}
\hline 1 & 2 & 3 \\
\hline 1 & 4 & \multicolumn{1}{|c}{} \\
\cline { 1 - 1 } 3 & 5 & \\
\cline { 1 - 1 } 4 & \multicolumn{2}{|c}{} \\
\cline { 1 - 1 }
\end{tabular}

Step 3. Place the entries removed from $P$ in Step 1 back into the Young tableau resulting from Step 2, in the same rows from which they were removed, to obtain

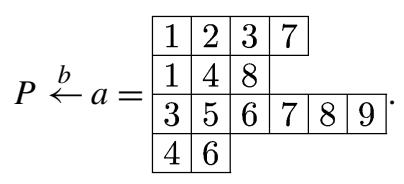

We define the bumping route of the bounded insertion algorithm to be the sequence of boxes in $P$ from which entries are bumped in Step 2, together with the new box which is added at the end of Step 2 .

Example 3.4 The bumping route in Example 3.3 is the set of boxes with •'s in the following Young diagram:

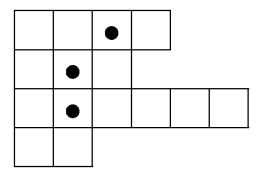

The new box is the lowest box containing a $\bullet$.

The bounded insertion algorithm is reversible: if $P \stackrel{b}{\leftarrow} a$ is computed, and we know $b$ and the location of the new box, then we can retrieve $P$ and $a$ by running the bounded insertion algorithm in reverse. Note that the reverse of Step 2 is the ordinary Schensted reverse insertion process. 
Suppose that $P$ is semistandard on $b$, that $a, a^{\prime}<b$, and that bounded insertion is performed twice in succession, resulting in $(P \stackrel{b}{\leftarrow} a) \stackrel{b}{\leftarrow} a^{\prime}$. Let $R$ and $B$ be the bumping route and new box of the first insertion, and let $R^{\prime}$ and $B^{\prime}$ be the bumping route and new box of the second insertion. We say that $R^{\prime}$ is weakly left of $R$ if for every box of $R$, there is a box of $R^{\prime}$ to the left of or equal to it; we say that $R$ is strictly left of $R^{\prime}$ if for every box of $R^{\prime}$, there is a box of $R$ to the left of it. We say that $B^{\prime}$ is strictly below $B$ if $B^{\prime}$ lies in a lower row than $B$; we say that $B$ is weakly below $B^{\prime}$ if $B$ lies in either the same row as $B^{\prime}$ or a lower row than $B^{\prime}$. The following Lemma is an immediate consequence of the analogous result for ordinary Schensted insertion (see [3]).

\section{Lemma 3.5}

(i) If $a \geq a^{\prime}$, then $R^{\prime}$ is weakly left of $R$ and $B^{\prime}$ is strictly below $B$.

(ii) If $a<a^{\prime}$, then $R$ is strictly left of $R^{\prime}$ and $B$ is weakly below $B^{\prime}$.

\section{Multisets on $\mathbb{N}$ and on $\mathbb{N}^{2}$}

Let $S$ be any set. A multiset $E$ on $S$ is defined to be a function $E: S \rightarrow\{0,1,2, \ldots$,$\} .$ One should think of $E$ as consisting of the set $S$ of elements, but with each $s \in S$ occurring $E(s)$ times. Note that a set is a special type of multiset in which each element occurs exactly once. Define the underlying set of $E$ to be $\{s \in S \mid E(s) \neq 0\}$, a subset of $S$. If $T$ is a subset of $S$, then we write $E \subset T$ if the underlying set of $E$ is a subset of $T$. We often write a multiset $E$ by listing its elements, $E=\left\{e_{1}, e_{2}, e_{3}, \ldots\right\}$, where the $e_{i}$ 's may not be distinct (in fact, each $e_{i}$ occurs $E\left(e_{i}\right)$ times in the list).

We call $E(s)$ the degree or multiplicity of $s$ in $E$. The multiset $E$ is said to be finite if $E(s)$ is nonzero for only finitely many $s \in S$. If $E$ is finite, then define the degree of $E$, denoted by $|E|$, to be the sum of $E(s)$ over all $s \in S$. If $E$ is not finite, then define the degree of $E$ to be $\infty$. Define the multisets $E \dot{U} F$ and $E \backslash F$ as follows:

$$
\begin{aligned}
(E \dot{\cup} F)(s) & =E(s)+F(s), \quad s \in S, \\
(E \backslash F)(s) & =\max \{E(s)-F(s), 0\}, \quad s \in S .
\end{aligned}
$$

Example 4.1 Let $E=\{a, b, b, b, c, c, c\}, F=\{b, b, c, d\}$. Then $|E|=7, E \dot{\cup} F=$ $\{a, b, b, b, b, b, c, c, c, c, d\}$, and $E \backslash F=\{a, b, c, c\}$.

\section{Multisets on $\mathbb{N}$}

Let $\mathbb{N}$ denote the positive integers. Let $E=\left\{e_{1}, e_{2}, \ldots\right\}$ be a multiset on $\mathbb{N}$, and let $z \in \mathbb{N}$. Define the multiset $E^{\leq z}:=\left\{e_{j} \in E \mid e_{j} \leq z\right\}$.

Let $A=\left\{a_{1}, a_{2}, \ldots\right\}$ and $B=\left\{b_{1}, b_{2}, \ldots\right\}$ be two multisets on $\mathbb{N}$ of the same degree, with $a_{i} \leq a_{i+1}, b_{i} \leq b_{i+1}$, for all $i$. We say that $A$ is less than or equal to $B$ in the termwise order if $a_{i} \leq b_{i}$ for all $i$, or equivalently if $\left|A^{<z}\right| \geq\left|B^{<z}\right|$ for all $z \in \mathbb{N}$. We denote this by $A \leq B$. We say that $A$ is less than $B$ in the strict termwise order if $a_{i}<b_{i}$ for all $i$. We denote this by $A \lessdot B$. Note that $\leq$ is a finer order than $\lessdot$. 
If $A, B, C$, and $D$ are multisets on $\mathbb{N}$ such that $|A \dot{\cup} D|=|B \dot{\cup} C|$, then we write

$$
A-C \leq B-D \text { to indicate that } A \dot{\cup} D \leq B \dot{\cup} C \text {. }
$$

Note that $A-B \leq C-D$ is a transitive relation.

In general no meaning is attached to the expression $A-C$ by itself. However, if $A$ and $C$ are both sets, then we may define $A-C:=A \dot{\cup}(\mathbb{N} \backslash C)$. If in addition $A \subset \bar{\beta}$ and $C \subset \beta$, then we may define $A-C:=A \dot{\cup}(\beta \backslash C)$. It is an easy check that both of these definitions are consistent with (1) (e.g., $A \dot{\cup}(\mathbb{N} \backslash C) \leq B \dot{\cup}(\mathbb{N} \backslash D)$ if and only if $A \dot{\cup} D \leq B \dot{\cup} C$ ).

Multisets on $\mathbb{N}^{2}$

Let $U=\left\{\left(e_{1}, f_{1}\right),\left(e_{2}, f_{2}\right), \ldots\right\}$ be a multiset on $\mathbb{N}^{2}$. Define $U_{(1)}$ and $U_{(2)}$ to be the multisets $\left\{e_{1}, e_{2}, \ldots\right\}$ and $\left\{f_{1}, f_{2}, \ldots\right\}$ respectively on $\mathbb{N}$. Define the nonvanishing, negative, and positive parts of $U$ to be the following multisets:

$$
\begin{aligned}
U^{\neq 0} & =\left\{\left(e_{i}, f_{i}\right) \in U \mid e_{i}-f_{i} \neq 0\right\}, \\
U^{-} & =\left\{\left(e_{i}, f_{i}\right) \in U \mid e_{i}-f_{i}<0\right\}, \\
U^{+} & =\left\{\left(e_{i}, f_{i}\right) \in U \mid e_{i}-f_{i}>0\right\} .
\end{aligned}
$$

It is useful to visualize the $e$-axis pointing downward and the $f$-axis pointing to the right, as illustrated below (the large squares cover the points of $\mathbb{N}^{2} \backslash\left(\mathbb{N}^{2}\right) \neq 0$ ):

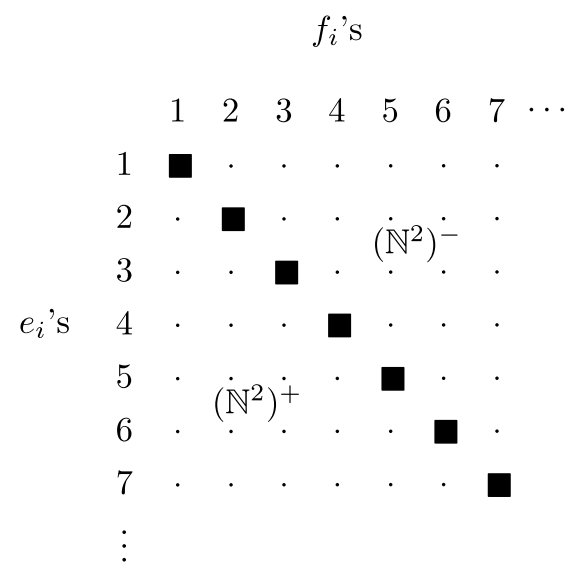

We say that $U$ is nonvanishing if $U \subset\left(\mathbb{N}^{2}\right)^{\neq 0}$, negative if $U \subset\left(\mathbb{N}^{2}\right)^{-}$, and positive if $U \subset\left(\mathbb{N}^{2}\right)^{+}$. Impose the following transitive relation on multisets on $\mathbb{N}^{2}$ :

$$
U \leq V \Longleftrightarrow U_{(1)}-U_{(2)} \leq V_{(1)}-V_{(2)}
$$

Let $\iota$ be the map on multisets on $\mathbb{N}^{2}$ defined by $\iota\left(\left\{\left(e_{1}, f_{1}\right),\left(e_{2}, f_{2}\right), \ldots\right\}\right)=$ $\left\{\left(f_{1}, e_{1}\right),\left(f_{2}, e_{2}\right), \ldots\right\}$. Then $\iota$ is an involution, and it maps negative multisets on 
$\mathbb{N}^{2}$ to positive ones and visa-versa. Thus $\iota$ is a bijective pairing between the sets of negative and positive multisets on $\mathbb{N}^{2}$. Note also that $U \leq V \Longleftrightarrow \iota(V) \leq \iota(U)$.

In this paper, all sets and multisets other than $\mathbb{N}, \mathbb{N}^{2}$, and multisets expressed explicitly as $A \dot{\cup}(\mathbb{N} \backslash C)$ where $A$ and $C$ are finite subsets of $\mathbb{N}$, are assumed to be finite.

\section{Semistandard notched bitableaux}

A notched bitableau is a pair $(P, Q)$ of notched tableaux of the same shape (i.e., the same number of rows and the same number of boxes in each row). The degree of $(P, Q)$ is the number of boxes in $P$ (or $Q$ ). A notched bitableau $(P, Q)$ is said to be row strict if both $P$ and $Q$ are row strict. A row strict notched bitableau $(P, Q)$ is said to be semistandard if

$$
P_{1}-Q_{1} \leq \cdots \leq P_{r}-Q_{r}
$$

A row strict notched bitableau $(P, Q)$ is said to be negative if $P_{i} \lessdot Q_{i}, i=1, \ldots, r$, positive if $P_{i}>Q_{i}, i=1, \ldots, r$, and nonvanishing if either

$$
P_{i} \lessdot Q_{i} \quad \text { or } \quad P_{i} \gg Q_{i},
$$

for each $i=1, \ldots, r$.

Example 5.1 Consider the notched bitableau

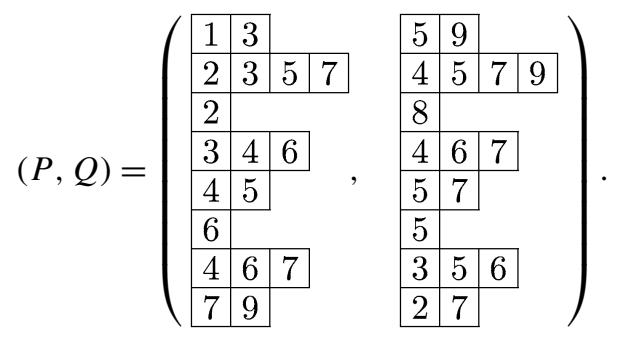

We have that

1. $(P, Q)$ is row strict.

2. $P_{1} \dot{\cup} Q_{2}=\{1,3,4,5,7,9\} \leq\{2,3,5,5,7,9\}=P_{2} \dot{\cup} Q_{1}$. Therefore, $P_{1}-Q_{1} \leq$ $P_{2}-Q_{2}$. Similarly, one checks that $P_{i}-Q_{i} \leq P_{i+1}-Q_{i+1}, i=2, \ldots, 7$. Thus $(P, Q)$ is semistandard.

3. $P_{i} \lessdot Q_{i}, i=1, \ldots, 5$, and $P_{i} \gg Q_{i}, i=6, \ldots, 8$. Thus $(P, Q)$ is nonvanishing.

Let $(P, Q)$ be a semistandard notched bitableau. If, for subsets $T$ and $W$ of $\mathbb{N}^{2}$,

$$
T_{(1)}-T_{(2)} \leq P_{1}-Q_{1} \quad \text { and } \quad P_{r}-Q_{r} \leq W_{(1)}-W_{(2)},
$$

then we say that $(P, Q)$ is bounded by $\mathbf{T}, \mathbf{W}$. Note that (5) combined with (3) implies

$$
T_{(1)}-T_{(2)} \leq P_{1}-Q_{1} \leq \cdots \leq P_{r}-Q_{r} \leq W_{(1)}-W_{(2)} .
$$


Thus (5) means that if $T_{(1)}$ and $T_{(2)}$ are placed above the top rows of $P$ and $Q$ respectively, and $W_{(1)}$ and $W_{(2)}$ are placed below the bottom rows of $P$ and $Q$ respectively, the resulting bitableau still satisfies (3).

Let $(P, Q)$ be a nonvanishing semistandard notched bitableau. Then all rows $\left(P_{i}, Q_{i}\right)$ of $(P, Q)$ for which $P_{i} \lessdot Q_{i}$ must lie above all rows $\left(P_{j}, Q_{j}\right)$ of $(P, Q)$ such that $P_{j}>Q_{j}$. Let $0 \leq i \leq r$ be maximal such that $P_{i} \lessdot Q_{i}$. Then the top $i$ rows of $P$ and $Q$ form a negative semistandard notched bitableau and the bottom $r-i$ rows of $P$ and $Q$ form a positive semistandard notched bitableau. These two bitableaux are called respectively the negative and positive parts of $(P, Q)$.

If $(P, Q)$ is a nonvanishing semistandard notched bitableau, define $\iota(P, Q)$ to be the notched bitableau obtained by reversing the order of the rows of $(Q, P)$. One checks that $\iota(P, Q)$ is a nonvanishing semistandard notched bitableau. The map $\iota$ is an involution, and it maps negative semistandard notched bitableaux to positive ones and visa-versa. Thus $\iota$ gives a bijective pairing between the sets of negative and positive semistandard notched bitableaux.

The definitions for semistandard notched tableaux and semistandard notched bitableaux appear to be quite different. The following Lemma gives a relationship between these two objects.

Lemma 5.2 Let $(P, Q)$ be a negative semistandard notched bitableau, and let $b$ be the minimum value of all entries of $Q$. Then $P$ is semistandard on $b$.

Proof Let $r$ be the number of rows of $P$. Suppose that, for some $i, 1 \leq i \leq r-1, P_{i+1}$ has exactly $x$ entries which are less than $b$, with $x>0$. We must show that (i) $P_{i}$ has at least $x$ entries which are less than $b$, and (ii) $\left\{P_{i, 1}, \ldots, P_{i, x}\right\} \leq\left\{P_{i+1,1}, \ldots, P_{i+1, x}\right\}$.

By (3),

$$
P_{i} \dot{\cup} Q_{i+1} \leq P_{i+1} \dot{\cup} Q_{i} .
$$

Therefore, since $P_{i+1} \dot{\cup} Q_{i}$ has (exactly) $x$ entries less than $b, P_{i} \dot{\cup} Q_{i+1}$ must have at least $x$ entries less than $b$, which must all be in $P_{i}$, since $b$ is the smallest entry of $Q$. This proves (i). The $x$ smallest entries of the left hand side and right hand side of (6) are $\left\{P_{i, 1}, \ldots, P_{i, x}\right\}$ and $\left\{P_{i+1,1}, \ldots, P_{i+1, x}\right\}$ respectively. Thus (6) implies (ii).

\section{The bounded RSK correspondence}

We next define the bounded RSK correspondence, BRSK, a function which maps negative multisets on $\mathbb{N}^{2}$ to negative semistandard notched bitableaux. Let $U=$ $\left\{\left(a_{1}, b_{1}\right), \ldots,\left(a_{t}, b_{t}\right)\right\}$ be a negative multiset on $\mathbb{N}^{2}$, whose entries we assume are listed in lexicographic order: (i) $b_{1} \geq \cdots \geq b_{t}$, and (ii) if for any $i \in\{1, \ldots, t-1\}$, $b_{i}=b_{i+1}$, then $a_{i} \geq a_{i+1}$. We inductively form a sequence of notched bitableaux $\left(P^{(0)}, Q^{(0)}\right),\left(P^{(1)}, Q^{(1)}\right), \ldots,\left(P^{(t)}, Q^{(t)}\right)$, such that $P^{(i)}$ is semistandard on $b_{i}$, $i=1, \ldots, t$, as follows:

Let $\left(P^{(0)}, Q^{(0)}\right)=(\emptyset, \emptyset)$, and let $b_{0}=b_{1}$. Assume inductively that we have formed $\left(P^{(i)}, Q^{(i)}\right)$, such that $P^{(i)}$ is semistandard on $b_{i}$, and thus on $b_{i+1}$, 
since $b_{i+1} \leq b_{i}$. Define $P^{(i+1)}=P^{(i)} \stackrel{b_{i+1}}{\longleftarrow} a_{i+1}$. Since bounded insertion preserves semistandardness on $b_{i+1}, P^{(i+1)}$ is also semistandard on $b_{i+1}$. Let $j$ be the row number of the new box of this bounded insertion. Define $Q^{(i+1)}$ to be the notched tableau obtained by placing $b_{i+1}$ on the left end of row $j$ of $Q^{(i)}$ (and shifting all other entries of $Q^{(i)}$ to the right one box). Clearly $P^{(i+1)}$ and $Q^{(i+1)}$ have the same shape.

Then $\operatorname{BRSK}(U)$ is defined to be $\left(P^{(t)}, Q^{(t)}\right)$. In the process above, we write $\left(P^{(i+1)}, Q^{(i+1)}\right)=\left(P^{(i)}, Q^{(i)}\right) \stackrel{b_{i+1}}{\leftarrow} a_{i+1}$. In terms of this notation,

$$
\operatorname{BRSK}(U)=\left((\emptyset, \emptyset) \stackrel{b_{1}}{\leftarrow} a_{1}\right) \cdots \stackrel{b_{t}}{\leftarrow} a_{t} .
$$

Example 6.1 Let $U=\{(7,8),(2,8),(6,7),(4,7),(1,7),(3,6),(2,4)\}$. Then

$$
\begin{aligned}
& P^{(0)}=\varnothing \\
& P^{(1)}=\emptyset \stackrel{8}{\leftarrow} 7=7 \\
& Q^{(0)}=\varnothing \\
& P^{(2)}=7 \stackrel{8}{\leftarrow 2} 2=\frac{2}{7} \\
& Q^{(1)}=8
\end{aligned}
$$

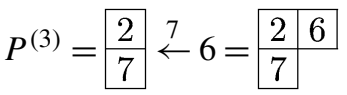

$$
\begin{aligned}
& Q^{(2)}=\begin{array}{l}
8 \\
8
\end{array}
\end{aligned}
$$

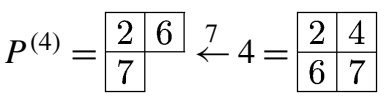

$$
\begin{aligned}
& Q^{(3)}=\begin{array}{|l|l|}
\hline 7 & 8 \\
\hline 8 &
\end{array} \\
& P^{(5)}=\begin{array}{|l|l|l|}
\hline 2 & 4 \\
\hline 6 & 7 \\
\hline
\end{array} \stackrel{2}{\leftarrow} 1=\begin{array}{|l|l|}
\hline 1 & 4 \\
\hline 2 & 7 \\
\hline 6 &
\end{array} \\
& Q^{(4)}=\begin{array}{|l|l|}
\hline 7 & 8 \\
\hline 7 & 8 \\
\hline
\end{array}
\end{aligned}
$$

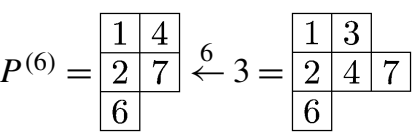

$$
\begin{aligned}
& Q^{(5)}=\begin{array}{|l|l|}
\hline 7 & 8 \\
\hline 7 & 8 \\
\hline 7 &
\end{array}
\end{aligned}
$$

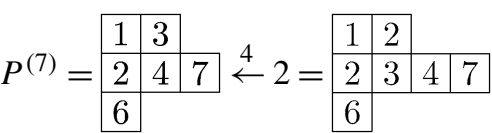

$$
\begin{aligned}
& Q^{(6)}=\begin{array}{|l|l|l|}
\hline 7 & 8 & \\
\hline 6 & 7 & 8 \\
\hline 7 & \multicolumn{2}{|l}{} \\
\cline { 1 - 1 }
\end{array}
\end{aligned}
$$

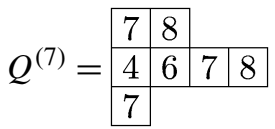

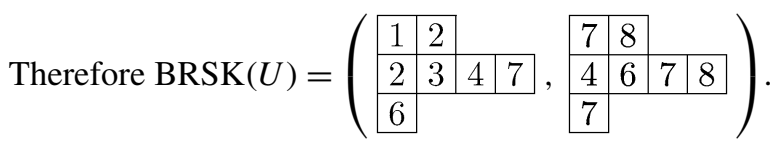

Lemma 6.2 If $U$ is a negative multiset on $\mathbb{N}^{2}$, then $\operatorname{BRSK}(U)$ is a negative semistandard notched bitableau.

Proof We use notation as in the definition of BRSK above. That $Q^{(i)}$ is row-strict for all $i$ follows from Lemma 3.5(i) and the fact that the entries of $U$ are listed in lexicographical order: if $b_{i+1}=b_{i}$ for some $i$, then since $a_{i+1} \leq a_{i}$, the new box of 
the $(i+1)$ st insertion must be strictly below the new box of $i$ th insertion. That $P^{(i)}$ is row strict and $\left(P^{(i)}, Q^{(i)}\right)$ is negative for all $i$ follows easily from the definition of BRSK, using induction. It remains to prove that $\left(P^{(i)}, Q^{(i)}\right)$ is semistandard for all $i$.

Let $P=P^{(i)}, Q=Q^{(i)}, P^{\prime}=P^{(i+1)}, Q^{\prime}=Q^{(i+1)}, a=a_{i+1}, b=b_{i+1}$, and assume inductively that $(P, Q)$ is a negative semistandard notched bitableau. Let $s$ be the number of rows of $P^{\prime}$ (and $Q^{\prime}$ ). We show that $\left(P^{\prime}, Q^{\prime}\right)$ satisfies (3), or equivalently, for any positive integer $z$,

$$
\left|\left(P_{j}^{\prime} \dot{\cup} Q_{j+1}^{\prime}\right)^{<z}\right| \geq\left|\left(P_{j+1}^{\prime} \dot{\cup} Q_{j}^{\prime}\right)^{<z}\right|, \quad j=1, \ldots, s-1 .
$$

By Lemma 5.2, $P$ is semistandard on $b$; hence so is $P^{\prime}$. Thus for $z \leq b$,

$$
\left|\left(P_{j}^{\prime} \dot{\cup} Q_{j+1}^{\prime}\right)^{<z}\right|=\left|\left(P_{j}^{\prime}\right)^{<z}\right| \geq\left|\left(P_{j+1}^{\prime}\right)^{<z}\right|=\left|\left(P_{j+1}^{\prime} \dot{\cup} Q_{j}^{\prime}\right)^{<z}\right|, j=1, \ldots, s-1 .
$$

Let $k$ be the row number of the new box (both in $P^{\prime}$ and $Q^{\prime}$ ) of this bounded insertion. Since $(P, Q)$ is semistandard, for $z>b, j \neq k-1$, and $j \neq k$,

$$
\left|\left(P_{j}^{\prime} \dot{\cup} Q_{j+1}^{\prime}\right)^{<z}\right|=\left|\left(P_{j} \dot{\cup} Q_{j+1}\right)^{<z}\right| \geq\left|\left(P_{j+1} \dot{\cup} Q_{j}\right)^{<z}\right|=\left|\left(P_{j+1}^{\prime} \dot{\cup} Q_{j}^{\prime}\right)^{<z}\right| .
$$

For $(z>b)$ and $(j=k-1$ or $j=k)$,

$$
\begin{aligned}
\left|\left(P_{j}^{\prime} \dot{\cup} Q_{j+1}^{\prime}\right)^{<z}\right| & =\left|\left(P_{j} \dot{\cup} Q_{j+1}\right)^{<z}\right|+1 \geq\left|\left(P_{j+1} \dot{\cup} Q_{j}\right)^{<z}\right|+1 \\
& =\left|\left(P_{j+1}^{\prime} \dot{\cup} Q_{j}^{\prime}\right)^{<z}\right| .
\end{aligned}
$$

Lemma 6.3 The map BRSK is a degree-preserving bijection from the set of negative multisets on $\mathbb{N}^{2}$ to the set of negative semistandard notched bitableaux.

Proof That BRSK is degree-preserving is obvious.

To show that BRSK is a bijection, we define its inverse, which we call the reverse of BRSK, or RBRSK.

Note that the bounded insertion used to form $\left(P^{(i+1)}, Q^{(i+1)}\right)$ from $\left(P^{(i)}, Q^{(i)}\right)$, $i=1, \ldots, t-1$, is reversible. In other words, by knowing only $\left(P^{(i+1)}, Q^{(i+1)}\right)$, we can retrieve $\left(P^{(i)}, Q^{(i)}\right), a_{i+1}$, and $b_{i+1}$. First, we obtain $b_{i+1}$; it is the minimum entry of $Q^{(i+1)}$. Then, in the lowest row in which $b_{i+1}$ appears in $Q^{(i+1)}$, select the greatest entry of $P^{(i+1)}$ which is less than $b_{i+1}$. This entry was the new box of the bounded insertion. If we begin reverse bounded insertion with this entry, we retrieve $P^{(i)}$ and $a_{i+1}$. Finally, $Q^{(i)}$ is retrieved from $Q^{(i+1)}$ by removing the lowest occurrence of $b_{i+1}$ appearing in $Q^{(i+1)}$. This occurrence must be on the left end of some row. All other entries of that row should be moved one box to the left, thus eliminating the empty box vacated by $b_{i+1}$.

It follows that we can reverse the entire sequence used to define BRSK by reversing each step in the sequence. If we generate $\left(P^{(t)}, Q^{(t)}\right)$ via BRSK, we can retrieve $U$ using this procedure. We will call the process of obtaining $\left(P^{(i-1)}, Q^{(i-1)}\right), a_{i}$, and $b_{i}$ from $\left(P^{(i)}, Q^{(i)}\right)$ described in the paragraph above a reverse step and denote it by $\left(P^{(i-1)}, Q^{(i-1)}\right)=\left(P^{(i)}, Q^{(i)}\right) \stackrel{b_{i}}{\rightarrow} a_{i}$. We will call the process of applying all the reverse steps sequentially to retrieve $U$ from $\left(P^{(t)}, Q^{(t)}\right)$ the reverse of BRSK, or 


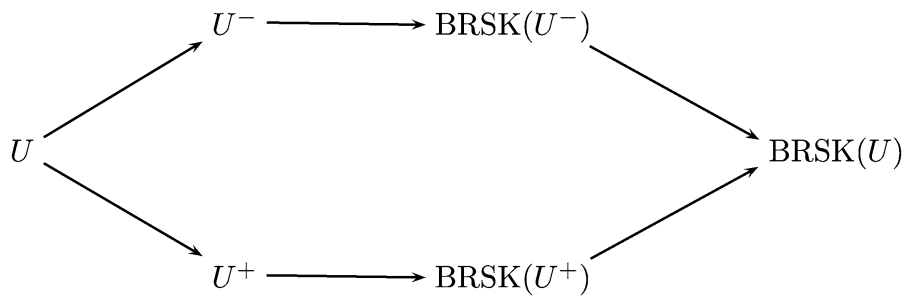

Fig. 1 The map BRSK

RBRSK. For example, if one applies RBRSK to the negative semistandard notched bitableau appearing on the bottom line of Example 6.1, one obtains the negative multiset $U$ from that example.

If $\left(P^{(t)}, Q^{(t)}\right)$ is an arbitrary semistandard notched bitableau (which we do not assume to be $\operatorname{BRSK}(U)$, for some $U)$, then we can still apply a sequence of reverse steps to $\left(P^{(t)}, Q^{(t)}\right)$, to sequentially obtain $\left(P^{(i)}, Q^{(i)}\right), a_{i}, b_{i}, i=t, \ldots, 1$. For this process to be well-defined, however, it must first be checked that the successive $\left(P^{(i)}, Q^{(i)}\right)$ are negative semistandard notched bitableaux. It suffices to prove a statement very similar to that proved in Lemma 6.2: if $(P, Q)$ is a negative semistandard notched bitableau, then $\left(P^{\prime}, Q^{\prime}\right):=(P, Q) \stackrel{b}{\rightarrow} a$ is a negative semistandard notched bitableau, $a<b$ are positive integers, and $b$ is less than or equal to all entries of $Q$. That $a<b$ are positive integers and $b$ is less than or equal to all entries of $Q$ follow immediately from the definition of a reverse step. That $\left(P^{\prime}, Q^{\prime}\right)$ is a negative semistandard notched bitableau follows in much the same manner as the proof of Lemma 6.2; we omit the details.

It remains to show that the elements $\left\{\left(a_{1}, b_{1}\right), \ldots,\left(a_{t}, b_{t}\right)\right\}$ of the negative multiset on $\mathbb{N}^{2}$ produced by applying this sequence of reverse steps to the arbitrary semistandard notched bitableau $\left(P^{(t)}, Q^{(t)}\right)$ are listed in lexicographic order. That $b_{i} \geq b_{i+1}$ follows from the definition of RBRSK: $b_{i+1}$ is the minimum entry of $Q^{(i+1)}$, which also has $b_{i}$ as an entry. If $b_{i}=b_{i+1}$, then $a_{i} \geq a_{i+1}$ is a consequence of Lemma 3.5(i) and (ii).

At each step, BRSK and the reverse of RBRSK are inverse to eachother. Thus they are inverse maps.

The map BRSK can be extended to all nonvanishing multisets on $\mathbb{N}^{2}$. If $U$ is a positive multisets on $\mathbb{N}^{2}$, then define $\operatorname{BRSK}(U)$ to be $\iota(\operatorname{BRSK}(\iota(U)))$. If $U$ is a nonvanishing multisets on $\mathbb{N}^{2}$, with negative and positive parts $U^{-}$and $U^{+}$, then define $\operatorname{BRSK}(U)$ to be the semistandard notched bitableau whose negative and positive parts are $\operatorname{BRSK}\left(U^{-}\right)$and $\operatorname{BRSK}\left(U^{+}\right)$(see Figure 1). As a consequence of Lemma 6.3, we obtain

Proposition 6.4 The map BRSK is a degree-preserving bijection from the set of nonvanishing (resp. negative, positive) multisets on $\mathbb{N}^{2}$ to the set of nonvanishing (resp. negative, positive) semistandard notched bitableaux.

The ordinary RSK correspondence is a degree-preserving bijection from the set of multisets on $\mathbb{N}^{2}$ to the set of semistandard bitableaux. The process used to define 
the bijection is similar to the process described above to define the BRSK. There are two essential differences between the two processes. First, in the ordinary RSK, the multiset is not first separated into its positive and negative parts. Indeed, the ordinary RSK is oblivious as to whether elements of the multiset are positive or negative. Secondly, in the ordinary RSK, ordinary Schensted insertion is used rather than bounded insertion. See [3] or [19] for more details on the ordinary RSK.

\section{Restricting the bounded RSK correspondence}

Thus far, there has been no reference to $\alpha, \beta$, or $\gamma$ in our definition or discussion of the bounded RSK. In fact, each of $\alpha, \beta$, and $\gamma$ is used to impose restrictions on the domain and codomain of the bounded RSK. It is the bounded RSK, with domain and codomain restricted according to $\alpha, \beta$, and $\gamma$, which is used in Section 8 to give geometrical information about $Y_{\alpha, \beta}^{\gamma}$.

In this section, we first show how $\beta$ restricts the domain and codomain of the bounded RSK. We then show how two subsets $T$ and $W$ of $\mathbb{N}^{2}, T$ negative and $W$ positive, restrict the domain and codomain of the bounded RSK. In Section 8, these two subsets will be replaced by $T_{\alpha}$ and $W_{\gamma}$, subsets of $\mathbb{N}^{2}$ determined by $\alpha$ and $\gamma$ respectively.

\section{Restricting by $\beta$}

Let $\beta \in I_{d, n}$. We say that a notched bitableau $(P, Q)$ is on $\bar{\beta} \times \beta$ if all entries of $P$ are in $\bar{\beta}$ and all entries of $Q$ are in $\beta$. It is clear from the construction of BRSK that if $U$ is a nonvanishing multiset on $\bar{\beta} \times \beta$, then $\operatorname{BRSK}(U)$ is a nonvanishing semistandard notched bitableau on $\bar{\beta} \times \beta$, and visa-versa. Thus, as a consequence of Corollary 6.4, we obtain

Corollary 7.1 The map BRSK restricts to a degree-preserving bijection from the set of nonvanishing (resp. negative, positive) multisets on $\bar{\beta} \times \beta$ to the set of nonvanishing (resp. negative, positive) notched bitableaux on $\bar{\beta} \times \beta$.

We remark that if $\beta$ is the largest or smallest element of $I_{d, n}(\{n-d+1, \ldots, n\}$ or $\{1, \ldots, d\}$ respectively), then the bounded RSK restricted to $\bar{\beta} \times \beta$ is the same algorithm as the ordinary RSK restricted to $\bar{\beta} \times \beta$.

Restricting by $T$ and $W$

A chain in $\mathbb{N}^{2}$ is a subset $C=\left\{\left(e_{1}, f_{1}\right), \ldots,\left(e_{m}, f_{m}\right)\right\}$ of $\mathbb{N}^{2}$ such that $e_{1}<\cdots<e_{m}$ and $f_{1}>\cdots>f_{m}$. Let $T$ and $W$ be negative and positive subsets of $\mathbb{N}^{2}$ respectively. A nonempty multiset $U$ on $\mathbb{N}^{2}$ is said to be bounded by $\mathbf{T}, \mathbf{W}$ if for every chain $C$ which is contained in the underlying set of $U$,

$$
T \leq C \leq W
$$

(where we use the order on multisets on $\mathbb{N}^{2}$ defined in Section 4). We note that, in general, this condition neither implies nor is implied by the condition $T \leq U \leq$ 
$W$. For special cases, a geometric interpretation in terms of a chain order for $U$ being bounded by $T, W$ appears in Sect. 9 (this interpretation is not necessary for our discussion here).

With this definition, the bounded RSK correspondence is a bounded function, in the sense that it maps bounded sets to bounded sets. More precisely, we have the following Lemma, whose proof appears in Sect. 11.

Lemma 7.2 If a nonvanishing multiset $U$ on $\mathbb{N}^{2}$ is bounded by $T, W$, then $\operatorname{BRSK}(U)$ is bounded by $T, W$.

Let $T$ and $W$ be negative and positive subsets of $\bar{\beta} \times \beta$, respectively. Combining Corollary 7.1 and Lemma 7.2, we obtain

Corollary 7.3 For any positive integer $m$, the number of degree $m$ nonvanishing multisets on $\bar{\beta} \times \beta$ bounded by $T, W$ is less than or equal to the number of degree $m$ nonvanishing semistandard notched bitableaux on $\bar{\beta} \times \beta$ bounded by $T, W$.

We mention that the converse of Lemma 7.2 is not a priori true, i.e., the reverse BRSK is not a priori bounded. Otherwise, we could state here that the two numbers in Corollary 7.3 are equal. In fact, the reverse BRSK is indeed bounded, but since we do not need this result for our purposes we omit the proof.

\section{A Gröbner basis}

We call $f=f_{\theta_{1}, \beta} \cdots f_{\theta_{r}, \beta} \in K\left[\mathcal{O}_{\beta}\right]$ a standard monomial if

$$
\theta_{1} \leq \cdots \leq \theta_{r}
$$

and for each $i \in\{1, \ldots, r\}$, either

$$
\theta_{i}<\beta \quad \text { or } \theta_{i}>\beta \text {. }
$$

If in addition, for $\alpha, \gamma \in I_{d, n}$,

$$
\alpha \leq \theta_{1} \quad \text { and } \quad \theta_{r} \leq \gamma,
$$

then we say that $f$ is standard on $Y_{\alpha, \beta}^{\gamma}$.

We remark that, in general, a standard monomial is not a monomial in the affine coordinates of $\mathcal{O}_{\beta}$ (the $x_{i j}$ 's); rather, it is a polynomial. It is only a monomial in the $f_{\theta, \beta}$ 's. Recall the following result (see [13]).

Theorem 8.1 The standard monomials on $Y_{\alpha, \beta}^{\gamma}$ form a basis for $K\left[Y_{\alpha, \beta}^{\gamma}\right]$.

We wish to give a different indexing set for the standard monomials on $Y_{\alpha, \beta}^{\gamma}$. Let $I_{\beta}$ be the pairs $(R, S)$ such that $R \subset \bar{\beta}, S \subset \beta$, and $|R|=|S|$. Defining $R-S:=$ $R \dot{\cup}(\beta \backslash S)$ (see Section 4), we have the following fact, which is easily verified:

The map $(R, S) \mapsto R-S$ is a bijection from $I_{\beta}$ to $I_{d, n}$. 
(Indeed, the inverse map is given by $\theta \mapsto(\theta \backslash \beta, \beta \backslash \theta)$ ). Thus, for instance, we may write a Plucker coordinate in $\mathbb{C}\left[\mathcal{O}_{\beta}\right]$ as $f_{R-S}$ instead of $f_{\theta, \beta}$. (In fact, $f_{R-S}$ is just plus or minus the minor of $\mathbb{C}\left[\mathcal{O}_{\beta}\right]$ with row set $R$ and column set $S$; in Example 2.1, $f_{\{1,4\}-\{2,7\}}=f_{\{1,4,5\}, \beta}$.)

Note that under this bijection, $(\emptyset, \emptyset)$ maps to $\beta$. Let $\left(R_{\alpha}, S_{\alpha}\right)$ and $\left(R_{\gamma}, S_{\gamma}\right)$ be the preimages of $\alpha$ and $\gamma$ respectively. Define $T_{\alpha}$ and $W_{\gamma}$ to be any subsets of $\bar{\beta} \times \beta$ such that $\left(T_{\alpha}\right)_{(1)}=R_{\alpha},\left(T_{\alpha}\right)_{(2)}=S_{\alpha},\left(W_{\gamma}\right)_{(1)}=R_{\gamma},\left(W_{\gamma}\right)_{(2)}=S_{\gamma}$.

Given any notched bitableau $(P, Q)$ which is on $\bar{\beta} \times \beta$, we can form the monomial $f=f_{P_{1}-Q_{1}} \cdots f_{P_{r}-Q_{r}} \in K\left[\mathcal{O}_{\beta}\right]$, where $P_{1}, \ldots, P_{r}$ are the rows of $P$ and $Q_{1}, \ldots, Q_{r}$ the rows of $Q$. Conversely, given any monomial of the form $f=$ $f_{P_{1}-Q_{1}} \cdots f_{P_{r}-Q_{r}} \in K\left[\mathcal{O}_{\beta}\right]$, where $P_{i}$ and $Q_{i}$ are subsets of $\bar{\beta}$ and $\beta$ respectively of the same cardinality, $i=1, \ldots, r$, we can form the notched bitableau $(P, Q)$ on $\bar{\beta} \times \beta$ whose rows are $\left(P_{1}, Q_{1}\right), \ldots,\left(P_{r}, Q_{r}\right)$. We have

$(P, Q)$ is semistandard, nonvanishing, and bounded by $T_{\alpha}, W_{\gamma}$

$$
\begin{aligned}
& \Longleftrightarrow(P, Q) \text { satisfies }(3),(4), \text { and (5) } \\
& \Longleftrightarrow\left(P_{1}, Q_{1}\right), \ldots,\left(P_{r}, Q_{r}\right) \text { satisfy (3), (4), and (5) } \\
& \Longleftrightarrow P_{1}-Q_{1}, \ldots, P_{r}-Q_{r} \text { satisfy (8), (9), and (10) } \\
& \Longleftrightarrow f_{P_{1}-Q_{1}} \cdots f_{P_{r}-Q_{r}} \text { is standard on } Y_{\alpha, \beta}^{\gamma} .
\end{aligned}
$$

When we write above that $\left(P_{1}, Q_{1}\right), \ldots,\left(P_{r}, Q_{r}\right)$ satisfy (3) and (5), we use (1) to describe the order. When we write that $P_{1}-Q_{1}, \ldots, P_{r}-Q_{r}$ satisfy (8) and (10), we use the termwise order on the $P_{i} \dot{\cup}\left(\beta \backslash Q_{i}\right)$. The equivalence of these orders is discussed in Section 4. To see that $\left(P_{i}, Q_{i}\right)$ satisfying (4) is equivalent to $P_{i}-Q_{i}$ satisfying (9), note that since $\bar{\beta} \cap \beta=\emptyset, P_{i} \cap Q_{i}=\emptyset$, and therefore $P_{i} \lessdot Q_{i} \Longleftrightarrow$ $P_{i}-Q_{i}=P_{i} \dot{\cup}\left(\beta \backslash Q_{i}\right)<\beta$.

This proves the following lemma.

Lemma 8.2 The degree $m$ nonvanishing semistandard notched bitableaux on $\bar{\beta} \times \beta$ bounded by $T_{\alpha}, W_{\gamma}$ form an indexing set for the degree $m$ standard monomials on $Y_{\alpha, \beta}^{\gamma}$.

As discussed in Section 2, the affine coordinates of $K\left[\mathcal{O}_{\beta}\right]$ are indexed by $\bar{\beta} \times \beta$. Thus monomials in the affine coordinates of $K\left[\mathcal{O}_{\beta}\right]$ are naturally indexed by multisets on $\bar{\beta} \times \beta$ : the monomial $x_{i_{1} j_{1}} \ldots x_{i_{t} j_{t}} \in K\left[\mathcal{O}_{\beta}\right]$ is indexed by the multiset $\left\{\left(i_{1}, j_{1}\right), \ldots,\left(i_{t}, j_{t}\right)\right\} \subset \bar{\beta} \times \beta$. Letting $U=\left\{\left(i_{1}, j_{1}\right), \ldots,\left(i_{t}, j_{t}\right)\right\} \subset \bar{\beta} \times \beta$, we shall denote $x_{i_{1} j_{1}} \ldots x_{i_{t} j_{t}}$ by $x_{U}$. Note that $x_{U}$ is square-free if and only if the multiset $U$ is in fact a set. We define a monomial order on $K\left[\mathcal{O}_{\beta}\right]$ as follows: first declare $x_{i j}<x_{i^{\prime} j^{\prime}}$ if $\left(i<i^{\prime}\right)$ or $\left(i=i^{\prime}\right.$ and $\left.j>j^{\prime}\right)$, then impose the lexicographic order on monomials. For our purposes, the critical feature of this monomial order is that the initial term of any minor $f_{R-S}$ is the Southwest-Northeast monomial of $f_{R-S}$ (when $f_{R-S}$ is written naturally as plus or minus a determinant all of whose entries are $x_{i j}$ 's). In other words, in $f_{R-S}=x_{C}$, where $C$ is the chain in $\bar{\beta} \times \beta$ with $C_{(1)}=R, C_{(2)}=S$ 
(in Example 2.1, in $\left.f_{\{1,4\}-\{2,7\}}=x_{17} x_{42}=x_{\{(1,7),(4,2)\}}\right)$. Any other monomial order with this property would also suit our purposes.

Lemma 8.3 The degree $m$ multisets on $\bar{\beta} \times \beta$ bounded by $T_{\alpha}, W_{\gamma}$ form an indexing set for the degree $m$ monomials of $K\left[\mathcal{O}_{\beta}\right] \backslash$ in $G_{\alpha, \beta}^{\gamma}$.

Proof

$$
\text { in } \begin{aligned}
G_{\alpha, \beta}^{\gamma} & \left.=\left\langle\text { in } f_{\theta, \beta}\right| \alpha \not \leq \theta \text { or } \theta \not \leq \gamma\right\rangle \\
& \left.=\left\langle\text { in } f_{R-S}\right| R_{\alpha}-S_{\alpha} \not \leq R-S \text { or } R-S \not \leq R_{\gamma}-S_{\gamma}\right\rangle \\
& \left.=\left\langle x_{C}\right| C \text { a chain, } R_{\alpha}-S_{\alpha} \not \leq C_{(1)}-C_{(2)} \text { or } C_{(1)}-C_{(2)} \not R_{\gamma}-S_{\gamma}\right\rangle \\
& \left.=\left\langle x_{C}\right| C \text { a chain, } T_{\alpha} \not \leq C \text { or } C \not \leq W_{\gamma}\right\rangle .
\end{aligned}
$$

Therefore,

$x_{U}$ is a monomial in $K\left[\mathcal{O}_{\beta}\right] \backslash$ in $G_{\alpha, \beta}^{\gamma}$

$\Longleftrightarrow x_{U}$ is not divisible by any $x_{C}, C$ a chain such that $T_{\alpha} \not \subset C$ or $C \not \leq W_{\gamma}$

$\Longleftrightarrow U$ contains no chains $C$ such that $T_{\alpha} \not \leq C$ or $C \not \leq W_{\gamma}$

$\Longleftrightarrow T_{\alpha} \leq C \leq W_{\gamma}$, for any chain $C$ in $U$

$\Longleftrightarrow U$ is bounded by $T_{\alpha}, W_{\gamma}$.

We are now ready to prove the main result of the paper.

Proof of Proposition 2.3 We wish to show that in $G_{\alpha, \beta}^{\gamma}=\operatorname{in}\left\langle G_{\alpha, \beta}^{\gamma}\right\rangle$. Since $G_{\alpha, \beta}^{\gamma} \subset$ $\left\langle G_{\alpha, \beta}^{\gamma}\right\rangle$, in $G_{\alpha, \beta}^{\gamma} \subset \operatorname{in}\left\langle G_{\alpha, \beta}^{\gamma}\right\rangle$. For any $m \geq 1$,

\# of degree $m$ monomials in $K\left[\mathcal{O}_{\beta}\right] \backslash$ in $G_{\alpha, \beta}^{\gamma}$

$\stackrel{a}{=}$ \# of degree $m$ multisets on $\bar{\beta} \times \beta$ bounded by $T_{\alpha}, W_{\gamma}$

$\stackrel{b}{\leq}$ \# of degree $m$ semistandard notched bitableaux on $\bar{\beta} \times \beta$ bounded by $T_{\alpha}, W_{\gamma}$

$\stackrel{c}{=}$ \# of degree $m$ standard monomials on $Y_{\alpha, \beta}^{\gamma}$

$\stackrel{d}{=}$ \# of degree $m$ monomials in $K\left[\mathcal{O}_{\beta}\right] \backslash$ in $\left\langle G_{\alpha, \beta}^{\gamma}\right\rangle$,

where $a$ follows from Lemma 8.3, $b$ from Corollary 7.3, $c$ from Lemma 8.2, and $d$ from the fact that standard monomials on $Y_{\alpha, \beta}^{\gamma}$ and the monomials in $K\left[\mathcal{O}_{\beta}\right] \backslash$ $\operatorname{in}\left\langle G_{\alpha, \beta}^{\gamma}\right\rangle$ both induce homogeneous bases for $K\left[\mathcal{O}_{\beta}\right] /\left\langle G_{\alpha, \beta}^{\gamma}\right\rangle$. Thus in $G_{\alpha, \beta}^{\gamma} \supset$ $\operatorname{in}\left\langle G_{\alpha, \beta}^{\gamma}\right\rangle$.

We point out that, as a consequence of this proof, inequality $b$ is actually an equality. 
Example 8.4 Let $n=6, d=3, \beta=\{3,5,6\}$. Then

$$
\mathcal{O}_{\beta}=\left\{\left(\begin{array}{ccc}
x_{13} & x_{15} & x_{16} \\
x_{23} & x_{25} & x_{26} \\
1 & 0 & 0 \\
x_{43} & x_{45} & x_{46} \\
0 & 1 & 0 \\
0 & 0 & 1
\end{array}\right), x_{i j} \in K\right\} .
$$

Let $\alpha=\{1,2,4\}, \gamma=\{4,5,6\}$. We list all objects identified with the monomial $x_{26} x_{45}^{2} x_{15} x_{13} x_{43} \in K\left[\mathcal{O}_{\beta}\right] \backslash$ in $G_{\alpha, \beta}^{\gamma}$ in the preceding discussion: (a) monomial in $K\left[\mathcal{O}_{\beta}\right] \backslash$ in $G_{\alpha, \beta}^{\gamma}$, (b) multiset on $\bar{\beta} \times \beta$ bounded by $T_{\alpha}, W_{\gamma}$, (c) semistandard notched bitableau on $\bar{\beta} \times \beta$ bounded by $T_{\alpha}, W_{\gamma}$, (d) standard monomial on $Y_{\alpha, \beta}^{\gamma}$, and (e) standard monomial on $Y_{\alpha, \beta}^{\gamma}$ (different indices).

(a) $x_{26} x_{45}^{2} x_{15} x_{13} x_{43}$

(b) $\{(2,6),(4,5),(4,5),(1,5),(1,3),(4,3)\}$

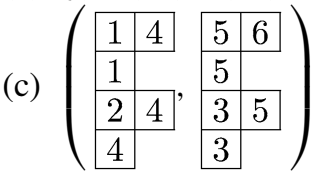

(d) $f_{\{1,4\}-\{5,6\}} f_{\{1\}-\{5\}} f_{\{2,4\}-\{3,5\}} f_{\{4\}-\{3\}}$

(e) $f_{\{1,3,4\}, \beta} f_{\{1,3,6\}, \beta} f_{\{2,4,6\}, \beta} f_{\{4,5,6\}, \beta}$.

Note that the semistandard notched tableau in (c) is obtained from the multiset in (b) by applying the BRSK.

Consider the following general Lemma on Gröbner Bases (see [2]).

Lemma 8.5 Let $R=K\left[x_{1}, \ldots, x_{m}\right]$ be a polynomial ring, let $I \subset R$ be a homogeneous ideal, and let $G=\left\{g_{1}, \ldots, g_{k}\right\}$ be a Gröbner basis for $I$, such that in $\left(g_{i}\right)$ is square-free, $i=1, \ldots, k$. Let $M$ be the maximum degree of a square-free monomial in $R \backslash \operatorname{in}(G)$. Then $\operatorname{dim}(R / I)=M$, and $\operatorname{deg}(R / I)$ is the number of square-free monomials of degree $M$ in $R \backslash \operatorname{in}(G)$.

Since the initial term of $f_{R-S}$ is square-free for any $(R, S) \in I_{\beta}$, Lemma 8.5 may be applied to our situation in order to obtain Corollary 2.4. Indeed, by Lemma 8.5, $\operatorname{deg}\left(Y_{\alpha, \beta}^{\gamma}\right)$ is the number of square-free monomials of degree $M$ in $K\left[\mathcal{O}_{\beta}\right] \backslash$ in $G_{\alpha, \beta}^{\gamma}$, where $M=\operatorname{dim}\left(Y_{\alpha, \beta}^{\gamma}\right)=l(\gamma)-l(\alpha)$. We remark that, in Lemma 8.5, we use the convention that 1 is the only square-free monomial of degree zero.

\section{Twisted chains and multiplicities}

The goal of this section and the next one is to establish Proposition 10.6, which also appears in [7-9], and [11]. Proposition 10.6 gives a combinatorial formula for multiplicities which involves counting families of nonintersecting lattice paths. Proposition 10.6 is essentially a reformulation of Corollary 2.4 in more combinatorial 
language. We establish Proposition 10.6 in two steps. In this section, we show that Corollary 2.4 implies Lemma 9.6; in Section 10, we show that Lemma 9.6 implies Proposition 10.6.

We begin by introducing twisted chains and chain boundedness, notions which allow us to place earlier results on combinatorial footing. We define the following partial orders on the negative elements of $\mathbb{N}^{2}$ : if $(e, f),(g, h) \in \mathbb{N}^{2}$, both negative, then

$$
\begin{array}{ll}
(e, f) \prec(g, h) & \text { if } f<h \text { and } e>g, \\
(e, f) \unlhd(g, h) & \text { if } f \leq h \text { and } e \geq g .
\end{array}
$$

Note that $\unlhd$ is a finer order than $\prec$. If $(c, d),(e, f) \in\left(\mathbb{N}^{2}\right)^{-}$, then define

$$
(c, d) \wedge(e, f)=(\max (c, e), \min (d, f)) \in \mathbb{N}^{2} .
$$

If $T=\left\{\left(e_{1}, e_{2}\right), \ldots,\left(e_{m}, e_{m+1}\right)\right\}$ is a subset of $\mathbb{N}^{2}$, then we say that $T$ is completely disjointed if $e_{i} \neq e_{j}$ when $i \neq j$. A negative twisted chain is a completely disjointed negative subset of $\mathbb{N}^{2}$ such that for any $u, v \in T, u \neq v$, either $u \prec v, v \prec u$, or $u \wedge v \notin\left(\mathbb{N}^{2}\right)^{-}$.

Example 9.1 A negative chain in $\mathbb{N}^{2}$, defined in Section 7 , can alternatively be described as a negative subset $\left\{u_{1}, \ldots, u_{m}\right\}$ of $\mathbb{N}^{2}$ such that $u_{1} \prec \cdots \prec u_{m}$. A negative chain is a negative twisted chain.

Let $T=\left\{\left(e_{1}, f_{1}\right), \ldots,\left(e_{m}, f_{m}\right)\right\}$ be a completely disjointed negative subset of $\mathbb{N}^{2}$ such that $f_{1}<\cdots<f_{t}$. For $\sigma \in S_{m}$, the permutation group on $m$ elements, we define $\sigma(T)=\left\{\left(e_{\sigma(1)}, f_{1}\right), \ldots,\left(e_{\sigma(m)}, f_{m}\right)\right\}$. Let $\mathcal{T}=\left\{\sigma(T) \mid \sigma \in S_{n}, \sigma(T)\right.$ negative $\}$. Impose the following total order on $\mathcal{T}$ : if $R=\left\{\left(a_{1}, f_{1}\right), \ldots,\left(a_{t}, f_{t}\right)\right\}$, $S=\left\{\left(b_{1}, f_{1}\right), \ldots,\left(b_{t}, f_{t}\right)\right\} \in \mathcal{T}$, then $R \stackrel{\text { lex }}{<} S$ if, for the smallest $i$ for which $a_{i} \neq b_{i}$, $a_{i}>b_{i}$. Since $\stackrel{\text { lex }}{<}$ is a total order, $\mathcal{T}$ has a unique minimal element, which we denote by $\widetilde{T}$.

Lemma 9.2 $\widetilde{T}$ is a negative twisted chain.

Proof Suppose that $\widetilde{T}=\left\{\left(c_{1}, f_{1}\right), \ldots,\left(c_{m}, f_{m}\right)\right\}$ is not a negative twisted chain. Then there exists $i<j$ such that $\left(c_{i}, f_{i}\right) \nprec\left(c_{j}, f_{j}\right),\left(c_{j}, f_{j}\right) \nprec\left(c_{i}, f_{i}\right)$, and $\left(c_{i}, f_{i}\right) \wedge\left(c_{j}, f_{j}\right) \in\left(\mathbb{N}^{2}\right)^{-}$. This implies that $c_{i}<c_{j}<f_{i}<f_{j}$. Letting $\sigma_{i, j}$ be the transposition which switches $i$ and $j$, we have that $\sigma_{i, j}(\widetilde{T})=\left\{\left(c_{1}, f_{1}\right), \ldots\right.$, $\left.\left(c_{j}, f_{i}\right), \ldots,\left(c_{i}, f_{j}\right), \ldots,\left(c_{m}, f_{m}\right)\right\}$. Since $c_{j}<f_{i}$ and $c_{i}<f_{j}, \sigma_{i, j}(\widetilde{T})$ is negative, and hence $\sigma_{i, j}(\widetilde{T}) \in \mathcal{T}$. The fact that $c_{i}<c_{j}$ implies that $\sigma_{i, j}(\widetilde{T}) \stackrel{\text { lex }}{<} \widetilde{T}$, which contradicts the minimality of $\widetilde{T}$.

Example 9.3 The set $\mathbb{N}^{2}$ is plotted in both (a) and (b) below. The large squares cover the points of $\mathbb{N}^{2} \backslash\left(\mathbb{N}^{2}\right)^{\neq 0}$, and thus separate the points of $\left(\mathbb{N}^{2}\right)^{-}$from those of $\left(\mathbb{N}^{2}\right)^{+}$. 
In (a), the $\times$ 's form a completely disjointed negative subset $T$ of $\mathbb{N}^{2}$. In (b), the $\times$ 's form $\widetilde{T}$. Note that $\widetilde{T}$ is a negative twisted chain, as required by Lemma 9.2.

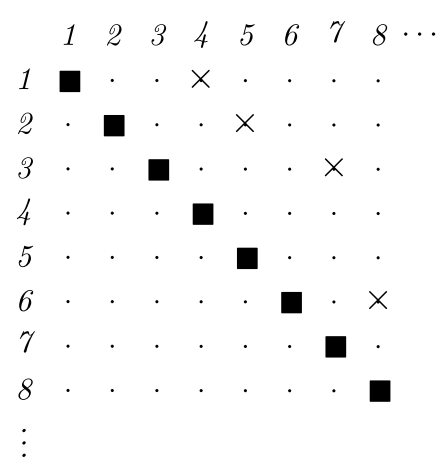

(a)

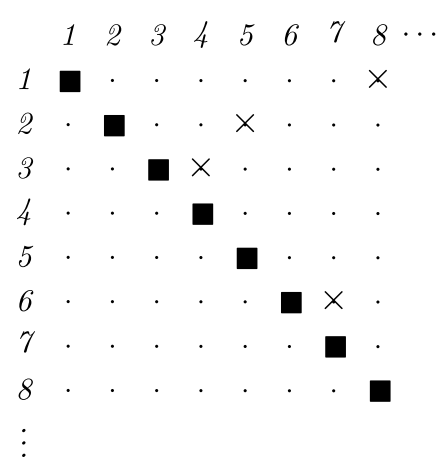

(b)

If $T$ is a positive subset of $\mathbb{N}^{2}$, then we say that $T$ is a positive twisted chain if $\iota(T)$ is a negative twisted chain. A twisted chain is a subset of $\mathbb{N}^{2}$ which is either a positive or a negative twisted chain.

For $R$ a negative subset of $\mathbb{N}^{2}$ and $x \in \mathbb{N}^{2}$ negative, define $\operatorname{depth}_{R}(x)$ to be the maximum $r$ such that there exists a chain $u_{1} \prec \cdots \prec u_{r}$ in $R$ with $u_{r} \unlhd x$. We extend $\unlhd$ to a transitive relation on subsets of $\mathbb{N}^{2}$ as follows. If $R, S$ are negative subsets of $\mathbb{N}^{2}$, then $R \unlhd S$ (or $S \unrhd R$ ) if $\operatorname{depth}_{R}(x) \geq \operatorname{depth}_{S}(x)$ for every negative $x \in \mathbb{N}^{2}$. Note that this is equivalent to $\operatorname{depth}_{R}(x) \geq \operatorname{depth}_{S}(x)$ for every $x \in S$. If $R, S$ are positive subsets of $\mathbb{N}^{2}$, then we say that $S \unrhd R$ if $\iota(S) \unlhd \iota(R)$. If $R$ is a negative subset of $\mathbb{N}^{2}$ and $S$ is a positive subset, then we say that $R \unlhd S$.

Recall the relation $\leq$ on multisets on $\mathbb{N}^{2}$ defined in Section 4 . The following Lemma, whose proof appears in Section 11, provides the key step in the proof of Lemma 9.6.

Lemma 9.4 Let $R$ and $S$ be twisted chains. Then $R \unlhd S \Longleftrightarrow R \leq S$.

Let $R$ and $S$ be negative and positive twisted chains respectively. We say that a multiset $U$ on $\mathbb{N}^{2}$ is chain-bounded by $\mathbf{R}, \mathbf{S}$ if $R \unlhd U^{-}$and $U^{+} \unlhd S$, or equivalently, if for every chain $C$ in $U$,

$$
R \unlhd C^{-} \quad \text { and } \quad C^{+} \unlhd S .
$$

In (7), one can replace $R \leq C \leq S$ by $R \leq C^{-}$and $C^{+} \leq S$. Thus, by Lemma 9.4, $U$ is chain-bounded by $R, S$ if and only if $U$ is bounded by $R, S$.

For the remainder of this section and the next one, we will be interested in twisted chains which are contained in $\bar{\beta} \times \beta$, a subset of $\mathbb{N}^{2}$. Example 9.5 illustrates two negative twisted chains in $\bar{\beta} \times \beta$. 
Example 9.5 The set $\bar{\beta} \times \beta \subset \mathbb{N}^{2}$, for $d=8, n=17, \beta=\{2,7,8,9,12,13,16,17\}$, is plotted below. The dotted line separates the negative from positive elements of $\bar{\beta} \times \beta$. The $\times$ 's form a negative twisted chain $R$ in $\bar{\beta} \times \beta$; the $\bullet$ 's form a negative twisted chain $S$ in $\bar{\beta} \times \beta$; and $R \unlhd S$.

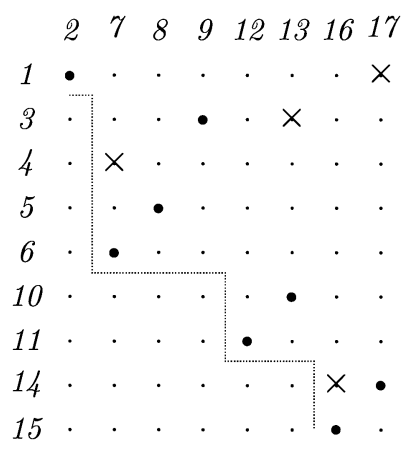

Lemma 9.6 Mult $_{e_{\beta}} X_{\alpha}^{\gamma}$ is the number of subsets $U$ of $\bar{\beta} \times \beta$ which are of maximal degree among those which are chain-bounded by $\widetilde{T}_{\alpha}, \widetilde{W_{\gamma}}$.

Proof Recall that if $U$ is a multiset on $\bar{\beta} \times \beta$, then the monomial $x_{U}$ is squarefree if and only if $U$ is a subset of $\bar{\beta} \times \beta$, i.e., each of its elements has degree 1 . By Corollary 2.4, Mult $e_{\beta} X_{\alpha}^{\gamma}$ is the number of square-free monomials of maximal degree in $K\left[\mathcal{O}_{\beta}\right] \backslash$ in $G_{\alpha, \beta}^{\gamma}$. By Lemma 8.3, this equals the number of subsets $U$ of $\bar{\beta} \times \beta$ which are of maximal degree among those bounded by $T_{\alpha}, W_{\gamma}$. However, a subset of $\bar{\beta} \times \beta$ is bounded by $T_{\alpha}, W_{\gamma}$ if and only if it is bounded by $\widetilde{T_{\alpha}}, \widetilde{W_{\gamma}}$ if and only if it is chain-bounded by $\widetilde{T_{\alpha}}, \widetilde{W_{\gamma}}$, where the last equivalence is due to Lemma 9.4.

\section{Path families and multiplicities}

For this section, we let $R$ and $S$ be fixed negative and positive twisted chains contained in $\bar{\beta} \times \beta$ respectively. Let

$$
\begin{aligned}
& \mathcal{M}_{R}=\max \left\{U \subset(\bar{\beta} \times \beta)^{-} \mid R \unlhd U\right\}, \\
& \mathcal{M}^{S}=\max \left\{V \subset(\bar{\beta} \times \beta)^{+} \mid V \unlhd S\right\}, \\
& \mathcal{M}_{R}^{S}=\max \left\{W \subset \bar{\beta} \times \beta \mid R \unlhd W^{-} \text {and } W^{+} \unlhd S\right\}
\end{aligned}
$$


where in each case by 'max' we mean the subsets $U, V$, or $W$ respectively of maximal degree. For example, $\mathcal{M}_{R}^{S}$ consists of the collection of subsets $W$ of $\bar{\beta} \times \beta$ which are of maximal degree among those which are chain-bounded by $R, S$. When $R=\widetilde{T}_{\alpha}$ and $S=\widetilde{W}_{\gamma}, \mathcal{M}_{R}^{S}$ consists precisely of the subsets $U$ of Lemma 9.6. In this section, in order to give a better formulation of Lemma 9.6 (see Proposition 10.6), we study the combinatorics of $\mathcal{M}_{R}^{S}$. Many of the definitions and ideas in this section are illustrated in Examples 10.7, 10.8, and 10.9.

Note that

$$
\mathcal{M}_{R}^{S}=\left\{U \dot{U} V \mid U \in \mathcal{M}_{R}, V \in \mathcal{M}^{S}\right\} .
$$

To study $\mathcal{M}_{R}^{S}$, we begin by considering $\mathcal{M}_{R}$, and thus restricting attention to negative subsets of $\bar{\beta} \times \beta$. We say that a subset $P \subset(\bar{\beta} \times \beta)^{-}$is depth-one if it contains no two-element chains. If $P$ is depth-one, then we say that it is a negative-path if the consecutive points are 'as close as possible' to each other, so that the points form a contiguous path on $(\bar{\beta} \times \beta)^{-}$which moves only down or to the right.

For $r=(e, f) \in(\bar{\beta} \times \beta)^{-}$, define

$$
\begin{aligned}
& \lfloor r\rfloor=\left(e, f^{\prime}\right), \text { where } f^{\prime}=\min \left\{y \in \beta \mid(e, y) \in(\bar{\beta} \times \beta)^{-}\right\}, \\
& \lceil r\rceil=\left(e^{\prime}, f\right), \text { where } e^{\prime}=\max \left\{x \in \bar{\beta} \mid(x, f) \in(\bar{\beta} \times \beta)^{-}\right\} .
\end{aligned}
$$

We form the path $P_{r}$, which begins at $\lfloor r\rfloor$, moves horizontally to $r$, then vertically to $\lceil r\rceil$. Note that since $R$ is a twisted chain, if $r^{\prime} \neq r$ then $P_{r^{\prime}} \cap P_{r}=\emptyset$. Furthermore, $R \unlhd \bigcup_{r \in R} P_{r}$. Define $d_{R}=\sum_{r \in R}\left|P_{r}\right|$. The following lemma is a straightforward consequence of the definitions.

Lemma 10.1 Let $Q$ be a depth-one negative subset of $\bar{\beta} \times \beta$ such that $P_{r} \unlhd Q$. Then $|Q| \leq\left|P_{r}\right|$, with equality if and only if $Q$ is a negative-path from $\lfloor r\rfloor$ to $\lceil r\rceil$.

If $U \subset(\bar{\beta} \times \beta)^{-}, R \unlhd U$, and $r \in R$, then define

$$
U_{R, r}:=\left\{u \in U \mid r \unlhd u, \operatorname{depth}_{U}(u)=\operatorname{depth}_{R}(r)\right\} .
$$

It follows from this definition that $U_{R, r}$ is depth-one. Indeed, if $u$ and $u^{\prime}$ are two elements of $U$ which form a chain, then without loss of generality $u \prec u^{\prime}$. Thus $\operatorname{depth}_{U}(u)<\operatorname{depth}_{U}\left(u^{\prime}\right)$, and in particular $\operatorname{depth}_{U}(u) \neq \operatorname{depth}_{U}\left(u^{\prime}\right)$. Thus $u$ and $u^{\prime}$ cannot both lie in $U_{R, r}$.

Lemma 10.2 Let $U \subset(\bar{\beta} \times \beta)^{-}$.

(i) If $R \unlhd U$, then $U=\dot{U}_{r \in R} U_{R, r}$.

(ii) If $R \unlhd U$, then $|U| \leq d_{R}$, with equality if and only if $U=\dot{U}_{r \in R} Q_{r}$, where $Q_{r}$ is a negative-path from $\lfloor r\rfloor$ to $\lceil r\rceil$.

(iii) Let $U=\bigcup_{r \in R} Q_{r} \subset(\bar{\beta} \times \beta)^{-}$, where $Q_{r}$ is a negative-path from $\lfloor r\rfloor$ to $\lceil r\rceil$. Then $R \unlhd U$. 
Proof (i) Let $u \in U$. Then since $R \unlhd U$, $\operatorname{depth}_{U}(u) \leq \operatorname{depth}_{R}(u)$. Thus $\operatorname{depth}_{U}(u)=$ $\operatorname{depth}_{R}(r)$ for some $r \unlhd u$. This proves that $U=\bigcup_{r \in R} U_{R, r}$. To prove that the union is disjoint, let $r, r^{\prime} \in R, r \neq r^{\prime}$, and let $v \in U_{R, r} \cap U_{R, r^{\prime}}$. By definition of $U_{R, r}$ and $U_{R, r^{\prime}}, \operatorname{depth}_{R}(r)=\operatorname{depth}_{U}(v)=\operatorname{depth}_{R}\left(r^{\prime}\right)$. Thus $r \nprec r^{\prime}$ and $r^{\prime} \nprec r$. Since $R$ is a twisted chain, $r \wedge r^{\prime} \notin\left(\mathbb{N}^{2}\right)^{-}$. But this implies that $v \notin\left(\mathbb{N}^{2}\right)^{-}$, a contradiction.

(ii) For each $u \in U_{R, r}, r \unlhd u$; thus since $U_{R, r}$ is depth-one, $P_{r} \unlhd U_{R, r}$. By Lemmas 10.1 and 10.2(i), $|U|=\left|\bigcup_{r \in R} U_{R, r}\right|=\sum_{r \in R}\left|U_{R, r}\right| \leq \sum_{r \in R}\left|P_{r}\right|=d_{R}$, with equality if and only if for all $r \in R, U_{R, r}$ is a negative-path from $\lfloor r\rfloor$ to $\lceil r\rceil$. We denote $U_{R, r}$ by $Q_{r}$.

(iii) For each $r \in R,\{r\} \unlhd Q_{r}$. Thus $R \unlhd \dot{U}_{r \in R} r \unlhd \dot{\bigcup}_{r \in R} Q_{r}=U$.

Lemma 10.2(ii) implies that any $U \in \mathcal{M}_{R}$ is a disjoint union $U=\bigcup_{r \in R} Q_{r}$, where $Q_{r}$ is a negative-path from $\lfloor r\rfloor$ to $\lceil r\rceil$. Lemma 10.2(iii) implies that any disjoint union $U=\cup_{r \in R} Q_{r}$, where $Q_{r}$ is a negative-path from $\lfloor r\rfloor$ to $\lceil r\rceil$, is an element of $\mathcal{M}_{r}$. Consequently we have

Corollary 10.3 $\mathcal{M}_{R}$ consists of the set of all possible disjoint unions $U=\dot{U}_{r \in R} Q_{r}$, where $Q_{r}$ is a negative-path from $\lfloor r\rfloor$ to $\lceil r\rceil$.

Similar analysis can be done on positive subsets of $\bar{\beta} \times \beta$. Here the notion of a positive-path is identical to that of a negative-path, except that it is contained in $(\bar{\beta} \times \beta)^{+}$instead of $(\bar{\beta} \times \beta)^{-}$. Likewise, the notions of $\lfloor s\rfloor$ and $\lceil s\rceil$ for $s \in(\bar{\beta} \times \beta)^{+}$ are defined analogously as for $s \in(\bar{\beta} \times \beta)^{-}$(see Example 10.7). We obtain

Corollary 10.4 $\mathcal{M}^{S}$ consists of the set of all possible disjoint unions $V=\bigcup_{s \in S} Q_{s}$, where $Q_{s}$ is a positive-path from $\lfloor s\rfloor$ to $\lceil s\rceil$.

The preceding two corollaries and (11) imply

Corollary 10.5 $\mathcal{M}_{R}^{S}$ consists of the set of all possible disjoint unions $W=$ $\dot{\cup}_{r \in R \cup S} Q_{r}$, where $Q_{r}$ is either a negative-path or a positive-path from $\lfloor r\rfloor$ to $\lceil r\rceil$, depending on whether $r$ is negative or positive.

The subsets $U$ of Lemma 9.6 are precisely the elements of $\mathcal{M}_{R}^{S}$, when $R=\widetilde{T}_{\alpha}$ and $S=\widetilde{W}_{\gamma}$. Therefore combining Lemma 9.6 and Corollary 10.5, we obtain

Proposition 10.6 Mult $e_{\beta} X_{\alpha}^{\gamma}$ is the number of disjoint unions $\dot{\bigcup}_{r \in \widetilde{T}_{\alpha} \cup \widetilde{W}_{\beta}} P_{r}$, where $P_{r}$ is either a negative-path or a positive-path from $\lfloor r\rfloor$ to $\lceil r\rceil$, depending on whether $r$ is negative or positive. 
We call a disjoint union as in Proposition 10.6 a family of nonintersecting paths in $\bar{\beta} \times \beta$. Proposition 10.6 also appears in [7-9], and [11].

Example 10.7 Let $d=8, n=17, \alpha=\{1,2,3,5,6,8,11,14\}, \beta=\{2,7,8,9,12,13$, $16,17\}, \gamma=\{8,9,11,13,14,15,16,17\}$.

(a) The negative and positive twisted chains $\widetilde{T}_{\alpha}=\left\{r_{1}, \ldots, r_{6}\right\}$ and $\widetilde{W_{\gamma}}=$ $\left\{s_{1}, \ldots, s_{3}\right\}$ in $\bar{\beta} \times \beta$.

(b) The set of $\lfloor r\rfloor$ 's and $\lceil r\rceil$ 's, for all $r \in \widetilde{T_{\alpha}} \cup \widetilde{W_{\gamma}}$. Note that $\left\lfloor r_{4}\right\rfloor=\left\lceil r_{4}\right\rceil=r_{4}$ and $\left\lfloor r_{5}\right\rfloor=\left\lceil r_{5}\right\rceil=r_{5}$.

(c), (d) Two families of nonintersecting paths from $\lfloor r\rfloor$ to $\lceil r\rceil, r \in \widetilde{T}_{\alpha} \cup \widetilde{W_{\gamma}}$. Mult $_{e_{\beta}} X_{\alpha}^{\gamma}$ is the number of such families. Note that the path family in (c) consists of the paths $\left\{P_{r} \mid r \in \widetilde{T_{\alpha}} \cup \widetilde{W_{\gamma}}\right\}$.

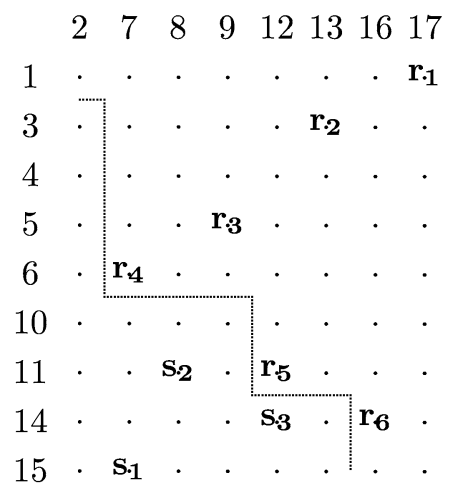

(a)

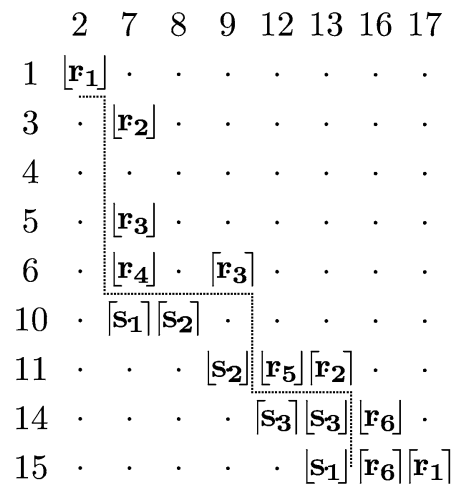

(b)

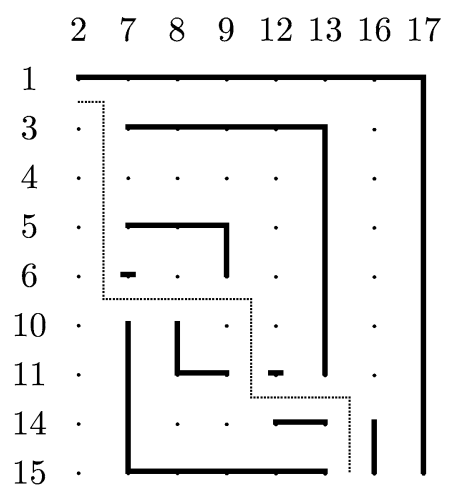

(c)

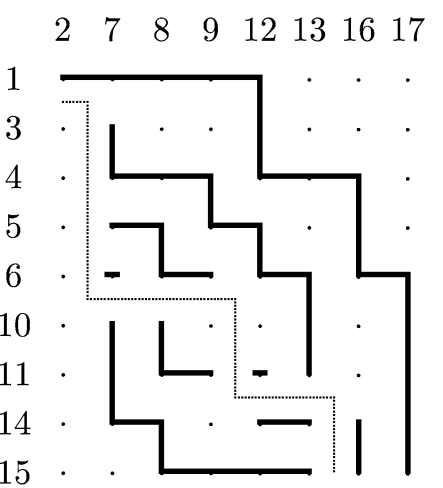

(d) 
Example 10.8 Let $d=4, n=9, \alpha=\{1,2,3,5\}, \beta=\{1,5,6,8\}, \gamma=\{3,6,8,9\}$.

We compute Mult $e_{\beta} X_{\alpha}^{\gamma}$. The following two diagrams show the negative and positive twisted chains $\widetilde{T}_{\alpha}=\left\{r_{1}, r_{2}\right\}$ and $\widetilde{W_{\gamma}}=\left\{s_{1}, s_{2}\right\}$ in $\bar{\beta} \times \beta$; and the set of $\lfloor r\rfloor$ 's and $\lceil r\rceil$ 's, for all $r \in \widetilde{T}_{\alpha} \cup \widetilde{W_{\gamma}}$.
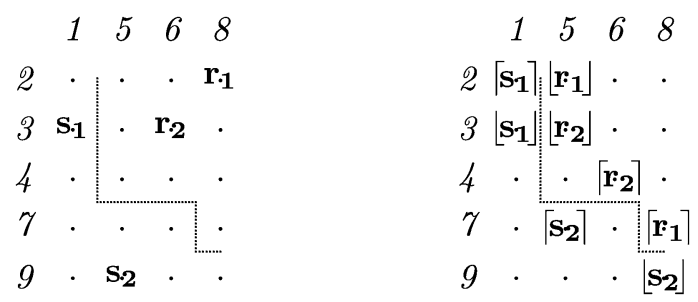

There are six nonintersecting path families from $\lfloor r\rfloor$ to $\lceil r\rceil, r \in \widetilde{T_{\alpha}} \cup \widetilde{W_{\gamma}}$, as shown below. Thus Mult $e_{\beta} X_{\alpha}^{\gamma}=6$.
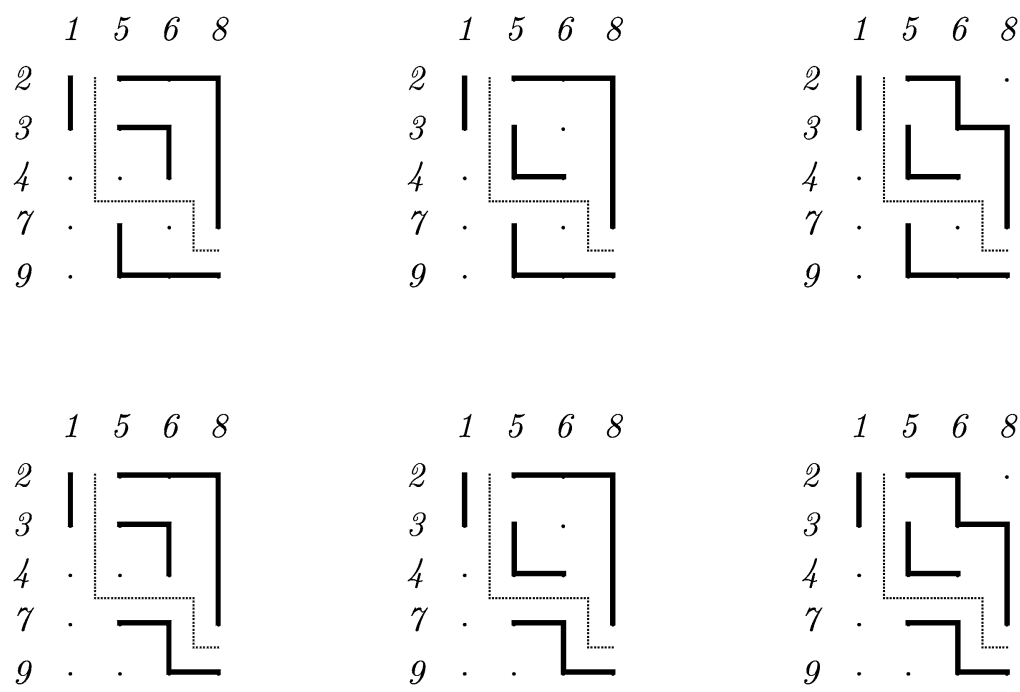

Example 10.9 Let $d=4, n=9, \alpha=\{1,2,3,5\}, \beta=\{1,5,6,8\}, \gamma=\{3,6,8,9\}$. We compute multiplicities at $e_{\beta}$ of the Schubert variety $X^{\gamma}$ and the opposite Schubert variety $X_{\alpha}$. Note that $\alpha, \beta$, and $\gamma$ are the same as in the previous example.

Observe that $X^{\gamma}=X_{\text {id }}^{\gamma}$ and $X_{\alpha}=X_{\alpha}^{\omega_{0}}$, where id $=\{1,2,3,4\}$ and $\omega_{0}=$ $\{6,7,8,9\}$. We have that $\widetilde{T_{\mathrm{id}}}=\left\{\sigma_{2,8}, \sigma_{3,6}, \sigma_{4,5}\right\}$ and $\widetilde{W_{\omega_{0}}}=\left\{\sigma_{9,1}, \sigma_{7,5}\right\}$, where $\sigma_{i, j}$ is the transposition exchanging $i$ and $j$. (Both $\widetilde{T_{\mathrm{id}}}$ and $\widetilde{W_{\omega_{0}}}$ are in fact chains.) 
There are two nonintersecting path families from $\lfloor r\rfloor$ to $\lceil r\rceil, r \in \widetilde{T_{\mathrm{id}}} \cup \widetilde{W_{\gamma}}$, as shown below. Thus Mult $e_{\beta} X^{\gamma}=$ Mult $_{e_{\beta}} X_{\text {id }}^{\gamma}=2$.
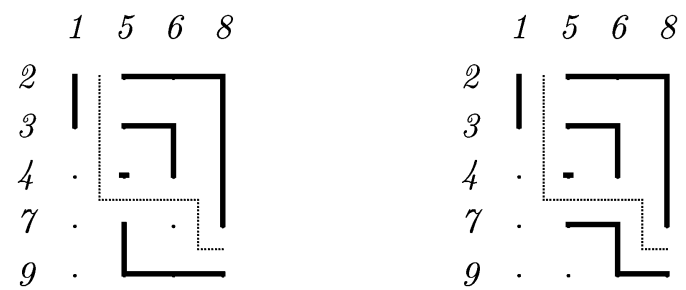

There are three nonintersecting path families from $\lfloor r\rfloor$ to $\lceil r\rceil, r \in \widetilde{T}_{\alpha} \cup \widetilde{W_{\omega_{0}}}$, as shown below. Thus Mult $e_{\beta} X_{\alpha}=\operatorname{Mult}_{e_{\beta}} X_{\alpha}^{\omega_{0}}=3$.
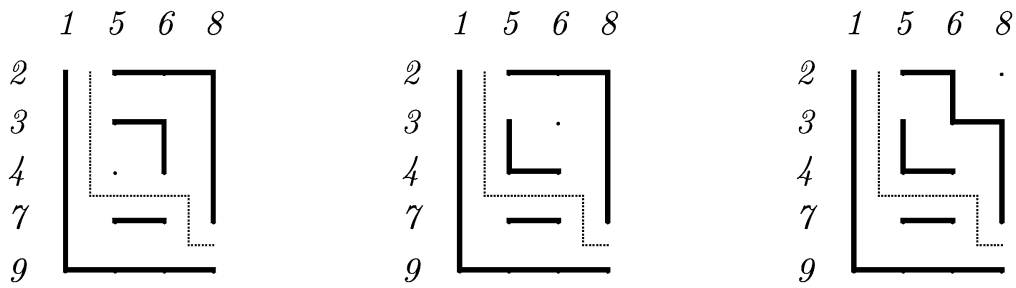

From this example and the previous one, we see that Mult $e_{\beta} X_{\alpha}^{\gamma}$ equals the product Mult $_{e_{\beta}} X^{\gamma} \cdot$ Mult $_{e_{\beta}} X_{\alpha}$. It is not difficult to show that this equality holds in general. This fact is also proven in [13], using different methods.

\section{Proofs}

In this section, we give proofs of Lemmas 7.2 and 9.4.

\section{Proof of Lemma 7.2}

Let $U$ be a nonvanishing multiset on $\mathbb{N}^{2}$, and let $T$ and $W$ be negative and positive subsets of $\mathbb{N}^{2}$ respectively with the property that $T_{(1)}, T_{(2)}, W_{(1)}$, and $W_{(2)}$ are both subsets of $\mathbb{N}$, i.e., multisets such that each value has cardinality one. Lemma 7.2 is part (v) of the following.

\section{Lemma 11.1}

(i) Suppose that $U=\left\{\left(a_{1}, b_{1}\right), \ldots,\left(a_{t}, b_{t}\right)\right\}$ is a negative multiset on $\mathbb{N}^{2}$ whose entries are listed in lexicographic order. For $k=1, \ldots, t$, let $U^{(k)}:=\left\{\left(a_{1}, b_{1}\right), \ldots\right.$, $\left.\left(a_{k}, b_{k}\right)\right\}$, and let $\left(P^{(k)}, Q^{(k)}\right)=\operatorname{BRSK}\left(U^{(k)}\right)$ (note that $\left(P^{(t)}, Q^{(t)}\right)=$ $\operatorname{BRSK}(U))$. Define $\left\{p_{1}^{(k)}, \ldots, p_{c_{k}}^{(k)}\right\}$ to be the first row of $P^{(k)}$ and $\left\{q_{1}^{(k)}, \ldots, q_{c_{k}}^{(k)}\right\}$ the first row of $Q^{(k)}$, both listed in increasing order. Let $m(k):=\max \{m \in$ $\left.\left\{1, \ldots, c_{k}\right\} \mid p_{m}^{(k)}<q_{1}^{(k)}\right\}=\left|\left(P_{1}^{(k)}\right)^{<q_{1}^{(k)}}\right|$. Then for $1 \leq j \leq m(k)$, there exists a 
chain $C_{k, j}$ in $U^{(k)}$ which has $j$ elements, the last of which has first component $p_{j}^{(k)}$.

(ii) If $U$ is bounded by $T, \emptyset$, then $\left(P^{(k)}, Q^{(k)}\right)$ is bounded by $T, \emptyset, k=1, \ldots, t$.

(iii) If $U$ is bounded by $T, \emptyset$, then $\operatorname{BRSK}(U)$ is bounded by $T, \emptyset$.

(iv) If $U$ is bounded by $\emptyset, W$, then $\operatorname{BRSK}(U)$ is bounded by $\emptyset, W$.

(v) If $U$ is bounded by $T, W$, then $\operatorname{BRSK}(U)$ is bounded by $T, W$.

Proof We prove (i) and (ii) together by induction on $k$, with $k=1$ the starting point for the induction. This case is technically covered by Case 2 below, although it can be checked quite easily: $U^{(1)}=\left\{\left(a_{1}, b_{1}\right)\right\}, P^{(1)}$ contains the sole entry $a_{1}$, and $Q^{(1)}$ contains the sole entry $b_{1}$. For (i), $m(1)=1, C_{1,1}=\left(a_{1}, b_{1}\right)$. For (ii), $U$ bounded by $T, \emptyset$ implies $U^{(1)}$ bounded by $T, \emptyset$, which is clearly equivalent to $\left(P^{(1)}, Q^{(1)}\right)$ bounded by $T, \emptyset$.

Let $k \in 2, \ldots, t-1$. Let $(P, Q)=\left(P^{(k)}, Q^{(k)}\right), a=a_{k+1}, b=b_{k+1},\left(P^{\prime}, Q^{\prime}\right)=$ $\left(P^{(k+1)}, Q^{(k+1)}\right), U=U^{(k)}, U^{\prime}=U^{(k+1)},\left\{p_{1}, \ldots, p_{c}\right\}=\left\{p_{1}^{(k)}, \ldots, p_{c_{k}}^{(k)}\right\},\left\{q_{1}, \ldots\right.$, $\left.q_{c}\right\}=\left\{q_{1}^{(k)}, \ldots, q_{c_{k}}^{(k)}\right\}$. Note that $\left\{p_{1}, \ldots, p_{c}\right\} \subset\left\{a_{1}, \ldots, a_{k}\right\},\left\{q_{1}, \ldots, q_{c}\right\} \subset\left\{b_{1}, \ldots\right.$, $\left.b_{k}\right\}$. Thus since $b$ is less than or equal to all elements of $\left\{b_{1}, \ldots, b_{k}\right\}, a<b \leq q_{1}$. We assume inductively that

$$
T_{(1)}-T_{(2)} \leq P_{1}-Q_{1},
$$

and prove that

$$
T_{(1)}-T_{(2)} \leq P_{1}^{\prime}-Q_{1}^{\prime}
$$

Equivalently, we prove that for all positive integers $z$,

$$
\left|\left(T_{(1)}-T_{(2)}\right)^{\leq z}\right| \geq\left|\left(P_{1}^{\prime}-Q_{1}^{\prime}\right)^{\leq z}\right|,
$$

where we use the definition $A-B:=A \dot{\cup}(\mathbb{N} \backslash B)$, where $A$ and $B$ are both subsets of $\mathbb{N}$ (see Sect. 4).

We consider two cases, corresponding to the two ways in which $\left(P_{1}^{\prime}, Q_{1}^{\prime}\right)$ can be obtained from $\left(P_{1}, Q_{1}\right)$.

Case 1. $P_{1}^{\prime}$ is obtained by a bumping $p_{l}$ in $P_{1}$, for some $1 \leq l \leq c$, i.e.,

$$
\begin{aligned}
& P_{1}^{\prime}=P_{1} \backslash\left\{p_{l}\right\} \cup \dot{\cup}\{a\}, \\
& Q_{1}^{\prime}=Q_{1}
\end{aligned}
$$

(i) The fact that $a$ bumps $p_{l}$ implies both $a \leq p_{l}$ and $p_{l}<b$. Hence $a \leq p_{l}<$ $b \leq q_{1}$, which implies $\left(P_{1}^{\prime}\right)^{<q_{1}}=\left(P_{1}\right)^{<q_{1}} \backslash\left\{p_{l}\right\} \cup\{a\}$. Thus $m(k+1)=m(k)$. For $j \in\{1, \ldots, m(k)\} \backslash\{l\}$, set $C_{k+1, j}=C_{k, j}$. If $l=1$ then set $C_{k+1, l}=\{(a, b)\}$. Otherwise, consider the chain $C_{k, l-1}=\left\{\left(g_{1}, h_{1}\right), \ldots,\left(p_{l-1}, h_{l-1}\right)\right\}$. Since $a$ bumps $p_{l}$, $a>p_{l-1}$. Thus $b<h_{l-1}$, since $(a, b)$ comes after $\left(p_{l-1}, h_{l-1}\right)$ in the ordered list of elements of $U^{\prime}$. Therefore $C:=C_{k, l-1} \cup\{(a, b)\}$ is a chain in $U^{\prime}$. We let $C_{k+1, l}$ be this chain.

(ii) For $z<a$ or $z \geq p_{l}$,

$$
\left|\left(T_{(1)}-T_{(2)}\right)^{\leq z}\right| \geq\left|\left(P_{1}-Q_{1}\right)^{\leq z}\right|=\left|\left(P_{1}^{\prime}-Q_{1}^{\prime}\right)^{\leq z}\right| .
$$


If $a=p_{l}$ then we are done. Thus we assume that $a<p_{l}$. We claim that for $a \leq z<$ $p_{l},\left|\left(C_{(1)}-C_{(2)}\right) \leq z\right|=\left|\left(P_{1}^{\prime}-Q_{1}^{\prime}\right) \leq z\right|$. Assuming the claim (and using the fact that $T \leq C \leq \emptyset$, since $U$ is bounded by $T, \emptyset)$ we have that for $a \leq z<p_{l}$,

$$
\left|\left(T_{(1)}-T_{(2)}\right)^{\leq z}\right| \geq\left|\left(C_{(1)}-C_{(2)}\right)^{\leq z}\right|=\left|\left(P_{1}^{\prime}-Q_{1}^{\prime}\right)^{\leq z}\right| .
$$

Now (12) and (13) prove the inductive step of (ii).

We now prove the claim. From the proof of (i), we have that $C=C_{k+1, l}=$ $\left\{\left(g_{1}, h_{1}\right), \ldots,\left(g_{l-1}, h_{l-1}\right),(a, b)\right\}$, where $g_{1}<\cdots<g_{l-1}<a<p_{l}<b<h_{l-1}<$ $\cdots<h_{1}$. Thus for $a \leq z<p_{l}$,

$$
\left|\left(C_{(1)}-C_{(2)}\right)^{\leq z}\right|=\left|\left(C_{(1)} \dot{\cup}\left(\mathbb{N} \backslash C_{(2)}\right)\right)^{\leq z}\right|=\left|\left(C_{(1)} \dot{\cup} \mathbb{N}\right)^{\leq z}\right|=l+z .
$$

Also, $p_{1}<\cdots<p_{l-1}<a<p_{l}<b \leq q_{1}<\cdots<q_{c}$. Thus for $a \leq z<p_{l}$,

$$
\left|\left(P_{1}^{\prime}-Q_{1}^{\prime}\right)^{\leq z}\right|=\left|\left(P_{1}^{\prime} \dot{\cup}\left(\mathbb{N} \backslash Q_{1}^{\prime}\right)\right)^{\leq z}\right|=\left|\left(P_{1}^{\prime} \dot{\cup} \mathbb{N}\right)^{\leq z}\right|=l+z
$$

Case 2. $P_{1}^{\prime}$ is obtained by adding $a$ to $P_{1}$ in position $l$ from the left and $Q_{1}^{\prime}$ is obtained by adding $b$ to the left end of $Q_{1}$ (and shifting all other entries of $Q_{1}$ to the right by one box), i.e.,

$$
\begin{aligned}
& P_{1}^{\prime}=P_{1} \dot{\cup}\{a\}, \\
& Q_{1}^{\prime}=Q_{1} \dot{\cup}\{b\} .
\end{aligned}
$$

(i) We have that $P_{1}^{\prime}=\left\{p_{1}, \ldots, p_{l-1}, a, p_{l}, \ldots, p_{c}\right\}, Q_{1}^{\prime}=\left\{b, q_{1}, \ldots, q_{c}\right\}$, where the elements of both sets are listed in strictly increasing order (note that $b<$ $q_{1}$ follows from the fact that $Q^{\prime}$ is row-strict, which is proven in Lemma 6.2). Now $p_{l-1}<a<b<q_{1}$ implies that $m(k) \geq l-1$. Note that $a<b \leq p_{l}$ (since $b>p_{l}$ would require that $a$ bump $p_{l}$ in the bounded insertion process). Thus $m(k+1)=l$. For $j \in\{1, \ldots, l-1\}$, set $C_{k+1, j}=C_{k, j}$. Consider the chain $C_{k, l-1}=$ $\left\{\left(g_{1}, h_{1}\right), \ldots,\left(g_{l-2}, h_{l-2}\right),\left(p_{l-1}, h_{l-1}\right)\right\}$. Now $a>p_{l-1}$, and this implies that $b<$ $h_{l-1}$, since $(a, b)$ comes after $\left(p_{l-1}, h_{l-1}\right)$ in the ordered list of elements of $U^{\prime}$. Therefore $C:=C_{k, l-1} \cup\{(a, b)\}$ is a chain in $U^{\prime}$. We let $C_{k+1, l}$ be this chain.

(ii) For $z<a$,

$$
\left|\left(T_{(1)}-T_{(2)}\right)^{\leq z}\right| \geq\left|\left(P_{1}-Q_{1}\right)^{\leq z}\right|=\left|\left(P_{1}^{\prime}-Q_{1}^{\prime}\right)^{\leq z}\right| .
$$

In fact, (14) holds for $z \geq b$ as well, since for such $z$,

$$
\begin{aligned}
\left|\left(P_{1}^{\prime}-Q_{1}^{\prime}\right)^{\leq z}\right| & =\left|\left(P_{1}^{\prime} \dot{\cup}\left(\mathbb{N} \backslash Q_{1}^{\prime}\right)\right)^{\leq z}\right| \\
& =\left|\left(P_{1}^{\prime}\right)^{\leq z}\right|+\left|\left(\mathbb{N} \backslash Q_{1}^{\prime}\right)^{\leq z}\right| \\
& =\left(\left|\left(P_{1}\right)^{\leq z}\right|+1\right)+\left(\left|\left(\mathbb{N} \backslash Q_{1}\right)^{\leq z}\right|-1\right) \\
& =\left|\left(P_{1}\right)^{\leq z}\right|+\left|\left(\mathbb{N} \backslash Q_{1}\right)^{\leq z}\right| \\
& =\left|\left(P_{1}-Q_{1}\right)^{\leq z}\right| .
\end{aligned}
$$

We claim that for $a \leq z<b,\left|\left(C_{(1)}-C_{(2)}\right) \leq z\right|=\left|\left(P_{1}^{\prime}-Q_{1}^{\prime}\right)^{\leq z}\right|$. Assuming the claim (and using the fact that $T \leq C \leq \emptyset$, since $U$ is bounded by $T, \emptyset$ ) we have that for 
$a \leq z<b$,

$$
\left|\left(T_{(1)}-T_{(2)}\right)^{\leq z}\right| \geq\left|\left(C_{(1)}-C_{(2)}\right)^{\leq z}\right|=\left|\left(P_{1}^{\prime}-Q_{1}^{\prime}\right)^{\leq z}\right| .
$$

Now (14) and (15) prove the inductive step of (ii).

We now prove the claim. From the proof of (i), we have that $C=C_{k+1, l}=$ $\left\{\left(g_{1}, h_{1}\right), \ldots,\left(g_{l-1}, h_{l-1}\right),(a, b)\right\}$, where $g_{1}<\cdots<g_{l-1}<a<b<h_{l-1}<\cdots<$ $h_{1}$. Thus for $a \leq z<b$,

$$
\left|\left(C_{(1)}-C_{(2)}\right)^{\leq z}\right|=\left|\left(C_{(1)} \dot{\cup}\left(\mathbb{N} \backslash C_{(2)}\right)\right)^{\leq z}\right|=\left|\left(C_{(1)} \dot{\cup} \mathbb{N}\right)^{\leq z}\right|=l+z
$$

Also, $p_{1}<\cdots<p_{l-1}<a<b \leq p_{l}, b<q_{1}<\cdots<q_{c}$. Thus for $a \leq z<b$,

$$
\left|\left(P_{1}^{\prime}-Q_{1}^{\prime}\right)^{\leq z}\right|=\left|\left(P_{1}^{\prime} \dot{\cup}\left(\mathbb{N} \backslash Q_{1}^{\prime}\right)\right)^{\leq z}\right|=\left|\left(P_{1}^{\prime} \dot{\cup} \mathbb{N}\right)^{\leq z}\right|=l+z .
$$

(iii) Set $k=t$ in (ii).

(iv) Use arguments similar to (i), (ii), and (iii), but for $U$ positive. Alternatively, one could apply the involution $\iota$ to (iii).

(v) Use (iii), (iv), and the fact that $U$ is bounded by $T, W$ if and only if $U^{-}$is bounded by $T, \emptyset$ and $U^{+}$is bounded by $\emptyset, W$; and similarly for $\operatorname{BRSK}(U)$.

\section{Proof of Lemma 9.4}

Parts (iii) and (iv) of the Lemma below imply Lemma 9.4. In this proof, for $R$ a subset of $\mathbb{N}^{2}$, we define $R_{(1)}-R_{(2)}$ to be the (infinite) multiset $R_{(1)} \dot{\cup} \mathbb{N} \backslash R_{(2)}$ (see Sect. 4).

\section{Lemma 11.2}

(i) Let $R, S$ be negative twisted chains. Then $R \unlhd S$ if and only if $\operatorname{depth}_{R}((z, z+$ $1)) \geq \operatorname{depth}_{S}((z, z+1))$ for all $z \in \mathbb{N}$.

(ii) Let $R$ be a negative twisted chain, and let $z \in \mathbb{N}$. Then $\operatorname{depth}_{R}(z, z+1)=$ $\left|\left(R_{(1)}-R_{(2)}\right) \leq z\right|-z$.

(iii) Let $R, S$ be negative twisted chains. Then $R \unlhd S \Longleftrightarrow R \leq S$.

(iv) Let $R, S$ be positive twisted chains. Then $R \unlhd S \Longleftrightarrow R \leq S$.

Proof (i) The "only if" direction is obvious. For $(e, f) \in\left(\mathbb{N}^{2}\right)^{-}$, define $D((e, f))=$ $\operatorname{depth}_{R}((e, f))-\operatorname{depth}_{S}((e, f))$. Suppose that $D((e, f))<0$ for some $(e, f) \in$ $\left(\mathbb{N}^{2}\right)^{-}$. We must show that $D((z, z+1))<0$ for some $z \in \mathbb{N}$. Suppose that $D((e, e+$ $1)) \geq 0$. Then $\operatorname{depth}_{R}((e, e+1))>\operatorname{depth}_{R}((e, f))$. Thus, there exists $\left(e^{\prime}, f^{\prime}\right) \in R$ such that $e^{\prime} \leq e$ and $e+1 \leq f^{\prime}<f$. Let $(g, h)$ be the one such with maximal $f^{\prime}$.

We claim that $\operatorname{depth}_{R}((h, h+1))=\operatorname{depth}_{R}((e, f))$. If not, then there exists $(p, q) \in R$ such that either (a) $e<p<h, h+1 \leq q$, (b) $p=h, h+1 \leq q$, or (c) $p \leq e, h+1 \leq q<f$. In case (a), $(p, q) \nprec(g, h),(g, h) \nprec(p, q)$, and $(g, h) \wedge(p, q)=(p, h) \in\left(\mathbb{N}^{2}\right)^{-}($since $p<h)$, contradicting the fact that $R$ is a negative twisted chain. In case (b), $p=h$, and thus $(g, h),(h, q) \in R$ contradicts the fact that $R$ is completely disjointed. In case (c), the maximality of $h$ is violated.

Since $(e, f) \unlhd(h, h+1), \operatorname{depth}_{S}((h, h+1)) \geq \operatorname{depth}_{S}((e, f))$. Thus $D((h, h+$ $1)) \leq D((e, f))<0$. 
(ii) Let $R=\left\{\left(e_{1}, f_{1}\right), \ldots,\left(e_{m}, f_{m}\right)\right\}$, with $e_{1}<\cdots<e_{m}$. Note that $\left\{\left(e_{i}, f_{i}\right) \in R \mid\right.$ $\left.e_{i} \leq z<z+1 \leq f_{i}\right\}$ consists of all the $\left(e_{i}, f_{i}\right) \in R$ such that $\left(e_{i}, f_{i}\right) \unlhd(z, z+1)$. Thus, since $R$ is a twisted chain, $\left\{\left(e_{i}, f_{i}\right) \in R \mid e_{i} \leq z<z+1 \leq f_{i}\right\}$ must form a chain. Hence depth $\operatorname{den}_{R}(z, z+1)=\left|\left\{\left(e_{i}, f_{i}\right) \in R \mid e_{i} \leq z<z+1 \leq f_{i}\right\}\right|$.

Recall that $R_{(1)}-R_{(2)}=\left\{e_{1}, \ldots, e_{m}\right\} \dot{\cup}\left(\mathbb{N} \backslash\left\{f_{1}, \ldots, f_{m}\right\}\right)$. The result now follows from the fact that

$$
\left|\left\{\left(e_{i}, f_{i}\right) \in R \mid e_{i} \leq z<z+1 \leq f_{i}\right\}\right|=\left|\left(R_{(1)}-R_{(2)}\right) \leq z \backslash \mathbb{N} \leq z\right| .
$$

To see why this equality holds, observe that $R_{(1)}-R_{(2)}$ can be obtained by starting with $\mathbb{N}$ and then successively replacing $f_{i}$ by $e_{i}, i=1, \ldots, m$. Such a replacement adds 1 to the number of elements less than or equal to $z$ if and only if $e_{i} \leq z$ and $f_{i} \geq z+1$ (and never subtracts 1 from the number of elements less than or equal to $z$, since $e_{i}<f_{i}$ ).

(iii)

$$
\begin{aligned}
R \unlhd S & \Longleftrightarrow \operatorname{depth}_{R}((z, z+1)) \geq \operatorname{depth}_{S}((z, z+1)), \quad z \in \mathbb{N} \\
& \Longleftrightarrow \mid\left(R_{(1)}-R_{(2)} \leq z|\geq|\left(S_{(1)}-S_{(2)} \leq z \mid, \quad z \in \mathbb{N}\right.\right. \\
& \Longleftrightarrow R_{(1)}-R_{(2)} \leq S_{(1)}-S_{(2)} \\
& \Longleftrightarrow R \leq S,
\end{aligned}
$$

where the first and second equivalences follow from (i) and (ii) respectively. (iv) is obtained by applying $\iota$ to both sides of (iii).

Acknowledgements I would like to thank A. Conca, V. Lakshmibai, P. Magyar, B. Sagan, and M. Shimozono for valuable discussions, suggestions, and corrections. I am also grateful to the referees for their careful readings and for pointing out a number of improvements and corrections.

\section{References}

1. Brion, M.: Lectures on the geometry of flag varieties. In: Topics in cohomological studies of algebraic varieties. Trends in Mathematics, pp. 33-85. Birkhäuser, Basel (2005)

2. Eisenbud, D.: Commutative Algebra. Graduate Texts in Mathematics, vol. 150. Springer, New York (1995)

3. Fulton, W.: Young Tableaux. London Mathematical Society Student Texts, vol. 35. Cambridge University Press, Cambridge (1997)

4. Ghorpade, S., Raghavan, K.N.: Hilbert functions of points on Schubert varieties in the symplectic Grassmannian. Trans. Am. Math. Soc. 358(12), 5401-5423 (2006)

5. Herzog, J., Trung, N.V.: Gröbner bases and multiplicity of determinantal and Pfaffian ideals. Adv. Math. 96(1), 1-37 (1992)

6. Hodge, W.V.D., Pedoe, D.: Methods of algebraic geometry, vol. I. Cambridge Mathematical Library. Cambridge University Press, Cambridge, 1994

7. Kodiyalam, V., Raghavan, K.N.: Hilbert functions of points on Schubert varieties in Grassmannians. J. Algebra 270(1), 28-54 (2003)

8. Krattenthaler, C.: On multiplicities of points on Schubert varieties in Grassmannians. Sém. Lothar. Comb. 45, Art. B45c (2000/2001) (electronic)

9. Krattenthaler, C.: On multiplicities of points on Schubert varieties in Grassmannians II. J. Algebr. Comb. 22, 273-288 (2005)

10. Kreiman, V.: Schubert classes in the equivariant K-theory and equivariant cohomology of the Grassmannian. Preprint arXiv:math.AG/0512204 
11. Kreiman, V.: Monomial bases and applications for Richardson and Schubert varieties in ordinary and affine Grassmannians. Ph.D. thesis, Northeastern University (2003)

12. Kreiman, V., Lakshmibai, V.: Multiplicities of singular points in Schubert varieties of Grassmannians. In: Algebra, Arithmetic and Geometry with Applications, West Lafayette, IN, 2000, pp. 553-563. Springer, Berlin (2004)

13. Kreiman, V., Lakshmibai, V.: Richardson varieties in the Grassmannian. In: Contributions to Automorphic Forms, Geometry, and Number Theory, pp. 573-597. Johns Hopkins University Press, Baltimore (2004)

14. Lakshmibai, V., Gonciulea, N.: Flag Varieties. Hermann, Paris (2001)

15. Lakshmibai, V., Raghavan, K.N., Sankaran, P.: Equivariant Giambelli and determinantal restriction formulas for the Grassmannian. Preprint arXiv:math.AG/0506015

16. Raghavan, K.N., Upadhyay, S.: Hilbert functions of points on Schubert varieties in the orthogonal Grassmannians. Preprint arXiv:0704.0542

17. Richardson, R.W.: Intersections of double cosets in algebraic groups. Indag. Math. (N.S.) 3(1), 69-77 (1992)

18. Rosenthal, J., Zelevinsky, A.: Multiplicities of points on Schubert varieties in Grassmannians. J. Algebr. Comb. 13(2), 213-218 (2001)

19. Sagan, B.E.: The Symmetric Group. Graduate Texts in Mathematics, vol. 203. Springer, New York (2001)

20. Stanley, R.P.: Some combinatorial aspects of the Schubert calculus. In: Combinatoire et représentation du groupe symétrique, Actes Table Ronde CNRS, Univ. Louis-Pasteur Strasbourg, Strasbourg, 1976. Lecture Notes in Mathematics, vol. 579, pp. 217-251. Springer, Berlin (1977)

21. Sturmfels, B.: Gröbner bases and Stanley decompositions of determinantal rings. Math. Z. 205(1), 137-144 (1990)

22. Viennot, G.: Une forme géométrique de la correspondance de Robinson-Schensted. In: Combinatoire et représentation du groupe symétrique, Actes Table Ronde CNRS, Univ. Louis-Pasteur Strasbourg, Strasbourg, 1976, Lecture Notes in Mathematics, vol. 579, pp. 29-58. Springer, Berlin (1977) 